\title{
A Stochastic Parameterization for Deep Convection Based on Equilibrium Statistics
}

\author{
R. S. Plant \\ Joint Centre for Mesoscale Meteorology, University of Reading, Reading, United Kingdom \\ G. C. CRAIG \\ Institut für Physik der Atmosphäre, DLR, Wessling, Germany
}

(Manuscript received 4 August 2006, in final form 18 April 2007)

\begin{abstract}
A stochastic parameterization scheme for deep convection is described, suitable for use in both climate and NWP models. Theoretical arguments and the results of cloud-resolving models are discussed in order to motivate the form of the scheme. In the deterministic limit, it tends to a spectrum of entraining/detraining plumes and is similar to other current parameterizations. The stochastic variability describes the local fluctuations about a large-scale equilibrium state. Plumes are drawn at random from a probability distribution function (PDF) that defines the chance of finding a plume of given cloud-base mass flux within each model grid box. The normalization of the PDF is given by the ensemble-mean mass flux, and this is computed with a CAPE closure method. The characteristics of each plume produced are determined using an adaptation of the plume model from the Kain-Fritsch parameterization. Initial tests in the single-column version of the Unified Model verify that the scheme is effective in producing the desired distributions of convective variability without adversely affecting the mean state.
\end{abstract}

\section{Introduction}

In numerical models of the atmosphere on global scales and mesoscales, the effects of moist convection cannot be adequately represented by the resolved-scale motions. Some form of parameterization scheme is necessary in order to obtain reliable and realistic results. Traditionally, such schemes are deterministic. The instantaneous grid-scale flow is taken as input and the scheme produces the feedbacks to that flow from the subgrid convective motions. In practice, there may, of course, be a wide range of subgrid states that are consistent with the resolved-scale flow, and therefore a deterministic scheme must be regarded conceptually as an attempt to evaluate the ensemble-mean effect of the subgrid states.

It is straightforward to demonstrate explicitly that the convective states consistent with a given resolved flow can indeed be wide ranging (see, e.g., $\mathrm{Xu}$ et al. 1992), given the lack of scale separation between the

Corresponding author address: R. S. Plant, Department of Meteorology, University of Reading, P.O. Box 243, Reading, Berkshire RG6 2BB, United Kingdom.

E-mail: r.s.plant@rdg.ac.uk resolved flow and the convective motions. Figure 1 shows the distribution of updraft mass fluxes (defined as in section 2) near cloud base that were obtained from a cloud-resolving-model (CRM) simulation of radiative-convective equilibrium. [The simulation was performed on a doubly periodic grid of $128 \times 128 \mathrm{~km}^{2}$ with a horizontal resolution of $2 \mathrm{~km}$. Convection was strongly forced by artificially cooling the troposphere at $16 \mathrm{~K} \mathrm{day}^{-1}$, with the sea surface temperature held fixed. The Coriolis parameter was set to zero and no mean shear was imposed. For full details of the simulation see Cohen (2001) and Cohen and Craig (2006).] The resulting mass fluxes are averaged over regions of different area, representative of possible grid box sizes in a larger-scale model. For such a strong and uniform external forcing, one might hope to find relatively little variability in the convective response after averaging over areas comparable with the grid box sizes of NWP or climate models. However, the actual distribution for a mesoscale grid length of $16 \mathrm{~km}$ is undeniably broad. The increased averaging for a grid length of $64 \mathrm{~km}$ does produce a narrower distribution. Nonetheless, its width is about $30 \%$ of the mean flux, indicating that fluctuations about the mean may still be a notable feature of the system. 


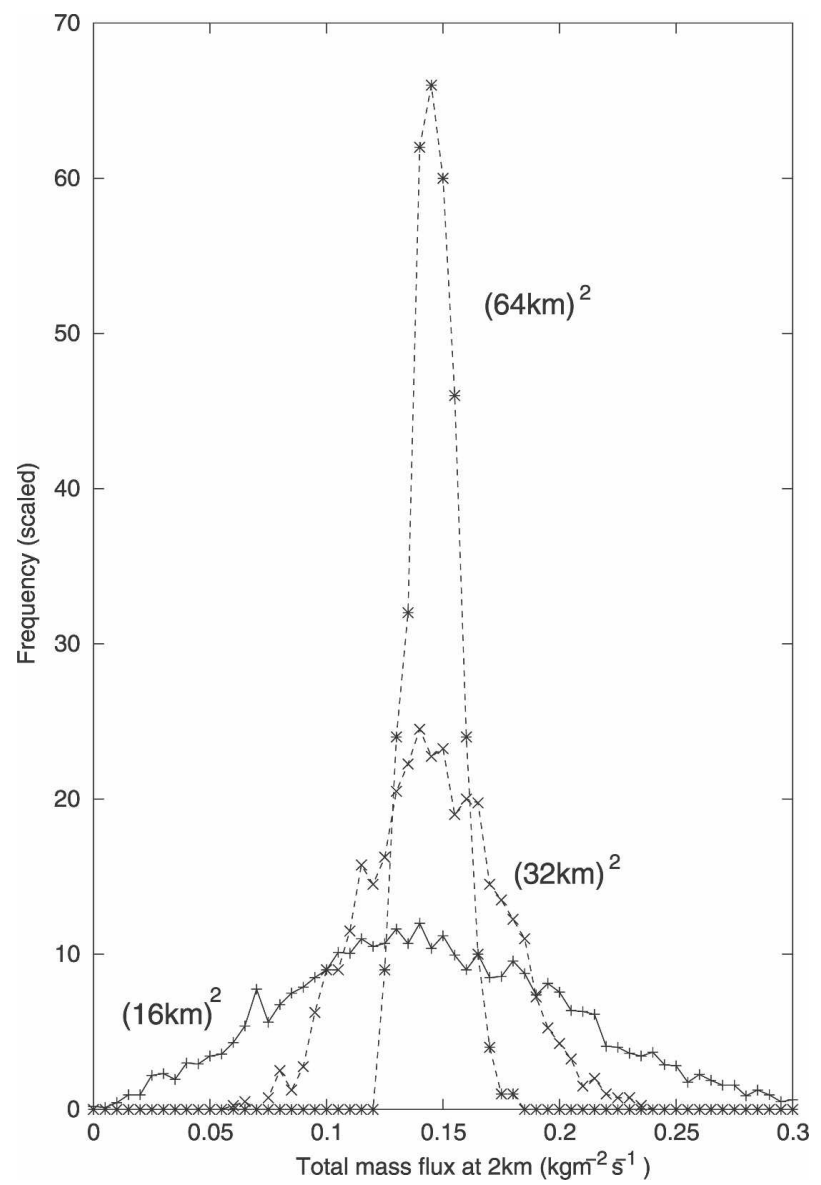

FIG. 1. Frequency plot of total convective mass flux per unit area (at a height of $2 \mathrm{~km}$ ) obtained from a CRM simulation (Cohen and Craig 2006) of radiative-convective equilibrium. The total mass flux is computed for different-sized areas and binned into intervals of $0.01 \mathrm{~kg} \mathrm{~m}^{-2} \mathrm{~s}^{-1}$. Data are taken from the established equilibrium state only using 340 times over 2 days. The vertical axis is scaled to account for the larger number of suitable areas that become available as the unit-averaging area is reduced in size.

Our discussion would be purely academic, were it not for the fact that convective fluctuations are capable of interacting strongly with nonlinearities in the convective system and with the resolved-scale dynamics. A powerful illustration of the point is provided by the marginal predictability of some convective structures on the mesoscale. In such cases, moist convection reacts strongly to near-grid-scale noise, which can cause simulations to evolve in quite distinct ways (Zhang et al. 2003; Done et al. 2006). Interactions of this sort contribute to the spread of ensembles that are based upon simulations with perturbed initial conditions. However, although existing ensemble techniques provide useful probabilistic information, in many situations the ensemble spread is insufficient to cover the full range of possible flows (Buizza 1997; Buizza et al. 2005). A not unrelated point is that many GCMs have insufficient high-frequency variability of convective heating (Ricciardulli and Garcia 2000) and precipitation (Horinouchi et al. 2003) in the tropics. This missing variability damages the model wave spectra in the middle atmosphere (Ricciardulli and Garcia 2000; Horinouchi et al. 2003) and so impacts on important low-frequency features of the climate system, such as the equatorial quasi-biennial oscillation (Horinouchi et al. 2003).

Current ensemble approaches usually aim to allow for uncertainties in the initial conditions and to do so in a controlled fashion, by which we mean that an increase in the number of ensemble members always leads to an increase in the information content. However, model uncertainty is not normally taken into account. Doubtless this is because details of such uncertainty are not known, the size and character of the errors involved being only sketchily understood.

Possible approaches include the construction of ensembles whose members take different parameter values in the parameterizations (Yang and Arritt 2002), employ different parameterizations entirely (Houtekamer et al. 1996; Stensrud et al. 2000; Bright and Mullen 2002), or even are derived from different models (Evans et al. 2000; Hou et al. 2001). Another approach has been to introduce a random multiplicative factor to the tendencies obtained from all parameterization schemes prior to feedback to the resolved scale (Buizza et al. 1999). Although such methods are not without flaws, nonetheless there is good evidence that there are genuine benefits from at least trying to deal with model uncertainty (Buizza et al. 1999; Hou et al. 2001; Mylne et al. 2002).

Progress can also be made by recognizing model uncertainties explicitly within the formulation of the model itself by introducing a stochastic forcing (Palmer 2001; Wilks 2005). It should be noted that there are some fundamental issues in the numerical solution of stochastic differential equations, but the situation is far from hopeless (Penland 2003; Ewald et al. 2004). The scheme of Buizza et al. (1999) represents perhaps the simplest and best-known example of a stochastic forcing. A straightforward improvement would be to scale the tendencies from each parameterization scheme separately (Lin and Neelin 2002; Teixeira and Reynolds 2008). However, perhaps a more promising approach is to introduce small-scale variability by including stochastic elements directly in a model's parameterization schemes. There are of course many possible ways of doing so. Existing examples include Palmer's (2001) suggestion of an approach using cellular automata and Bright and Mullen's (2002) use of a stochastic element 
in the trigger function of the Kain and Fritsch (1993) convection scheme. Alternatively, Majda and Khouider (2002) and Khouider et al. (2003) have described a stochastic scheme for evaluating the fractional area of a grid box that supports deep convection, while Lin and Neelin (2003) have proposed stochastic deep-convective parameterizations based on random perturbations to either the CAPE (Lin and Neelin 2000) or to the vertical heating profile.

The consensus emerging from the above studies is that the use of stochastic techniques to introduce smallscale variability to numerical models of the atmosphere is desirable for both conceptual and practical reasons (see also Williams 2006; Hermanson 2006). However, the stochastic schemes listed above are based on rather ad hoc assumptions about the time and space scales and structures of convective variability. This is not to deny their value. Indeed, there are good reasons for exploring different types of stochastic representations and their impacts with frameworks that are relatively straightforward. However, stochastic convection schemes should ultimately be based on systematic observations or simulations of convective behavior.

In this paper we describe a first attempt to build and test a stochastic cumulus parameterization that will, in a limited sense, produce the "correct" convective variability. In particular, the scheme is designed to reproduce the convective fluctuations that occur in radiativeconvective equilibrium over a uniform sea surface, a situation that is relatively well understood from theory and cumulus ensemble simulations (Craig and Cohen 2006; Cohen and Craig 2006).

Section 2 summarizes the key properties of convective variability in radiative-convective equilibrium (section 2a) and the implications for the design of a parameterization (section 2b). Sections 3 and 4 describe in detail the implementation of the scheme. Tests with a single-column model are described in section 5 to demonstrate the robustness of the scheme and its ability to produce correct behavior when interacting with an atmospheric state that can vary in time. It will also be important to demonstrate that the correct variability is reproduced in a full three-dimensional model with arbitrary grid size; this will be subject of a follow-up paper.

\section{Basis for a stochastic scheme}

In this paper we attempt to construct a stochastic parameterization based on a physical description of convective variability. In particular, we follow Arakawa and Schubert (1974) and assume the existence of a statistical equilibrium where the total mass flux of the en- semble of convective clouds found in a region is controlled by the large-scale environment. It is important to note that "large-scale environment" is defined by the dynamics of the meteorological situation and does not necessarily correspond to a region defined by the model grid length (although the grid length must be at least small enough to resolve the dynamical features). Arakawa and Schubert (1974) explained that the size of the region required to define an equilibrium must be large enough to contain many clouds-a statement that will be made more precise in the next section. A subregion, perhaps a model grid box, that is smaller than required will contain only a subset of the equilibrium convective ensemble. Its (spatially averaged) properties at any given moment will thus not equal those of the full ensemble and will vary in adjacent subregions, even though the system as a whole is in equilibrium. The mass flux in a subregion will thus be a random variable, but the fact that the convection is a subensemble of an equilibrium system implies that it will be drawn from a distribution determined by the large-scale flow.

The basic outline of an equilibrium-based parameterization that includes this randomness is as follows:

1) Average the atmospheric state (temperature, moisture, etc.) in the horizontal, over a region large enough to contain many clouds (possibly many grid boxes) to determine the large-scale environmental properties;

2) Compute the equilibrium statistics of the full convective ensemble;

3) Draw randomly from the equilibrium distribution to get the convective mass flux and other cumulus properties in each grid box;

4) Compute convective tendencies of grid box atmospheric variables like temperature and moisture from the mass flux and cumulus properties.

The crucial additional information required for the stochastic parameterization is the equilibrium distribution to be used in steps 2 and 3, and implicitly the scale required for spatial averaging in step 1 . A conventional mass-flux-based scheme only requires the mean mass flux in a grid box.

A model for equilibrium convective statistics was presented by Craig and Cohen (2006) and tested in numerical experiments (Cohen and Craig 2006), and will serve as the basis of the parameterization presented here. The next section (2a) summarizes this work, and the following section (2b) presents an explicit version of the above algorithm.

It should be recognized that the concept of a largescale environment is not well defined unless there exists 
a scale separation in the spectrum of cumulus dynamics. Whether any such separation exists has been questioned (e.g., Mapes 1997), most recently on the basis of evidence for $1 / f$ noise (Yano et al. 2001, 2004a) and self-organized criticality (Peters and Neelin 2006) in the tropics. Nonetheless, the concept is the basis for most, if not all current, cumulus parameterizations (Arakawa 2004).

\section{a. Fluctuations in radiative-convective equilibrium}

The convecting atmosphere is considered to support an ensemble of convective clouds (updrafts or updraftdowndraft pairs). Assuming a large-scale environment that is in equilibrium, there are two contributions to the convective variability in a subregion. First, the individual clouds may be weaker or stronger; that is, clouds have different mass fluxes. Second, a region of given area may contain a larger or smaller number of clouds, depending on where it is located.

Using elementary concepts from statistical mechanics, Craig and Cohen (2006) showed that for an ensemble of weakly interacting convective clouds in statistical equilibrium, the probability distribution function (PDF) of mass flux per cloud, $m$, is exponential:

$$
p(m) d m=\frac{1}{\langle m\rangle} \exp \left(\frac{-m}{\langle m\rangle}\right) d m,
$$

where angled brackets have been used to denote an ensemble average. The distribution has been verified in CRM simulations of radiative-convective equilibrium (Cohen 2001; Cohen and Craig 2006). The updraft mass fluxes in those CRM simulations were defined for the updraft cores: connected grid points, each with a vertical velocity larger than $1 \mathrm{~m} \mathrm{~s}^{-1}$ (LeMone and Zipser 1980). The mass flux was then $\rho A \bar{w}$, where $A$ is the area of the updraft core and the overbar denotes an average over contributing grid points. The relationship between this definition of mass flux and those used in a convective parameterization can be problematic (Yano et al. 2004b). Nonetheless, we shall assume that the same distribution [Eq. (1)] can also be used to describe the parameterized mass flux.

The PDF in Eq. (1) applies to a fixed level of the atmosphere. However, there is nothing in the arguments of Craig and Cohen (2006) to constrain what that level should be. For verification purposes, Cohen and Craig tested the distribution at $2.4 \mathrm{~km}$ (at the moist static energy minimum; their Figs. 1 and 2) and also just above cloud base. Further testing reveals the exponential shape to be remarkably robust, applying over a wide range of heights and with different forcings of the CRM. For example (other examples are given by Len- nard 2004), Fig. 2 shows histograms for tropospheric cooling rates of 8 and $16 \mathrm{~K} \mathrm{day}^{-1}$ at heights of 3.1 and $1.3 \mathrm{~km}$, respectively. (Note that the distribution does not extend to the smallest updrafts, a truncation that arises from the finite grid length and the cloud definition used.)

The assumptions leading to the exponential distribution include a statement of equilibrium that enables one to link the large-scale forcing to the ensemble-mean convective response. The strength of the response can be characterized by the ensemble-mean mass flux, $\langle M\rangle=\langle N\rangle\langle m\rangle$, where $\langle N\rangle$ is the ensemble-mean number of convective clouds present. In equilibrium $\langle M\rangle$ can be regarded as some function of processes operating on the large scale. Thus, we follow the standard practice in mass-flux schemes of regarding $\langle M\rangle$ as being defined by a closure assumption, specified in section 4 .

Individual clouds within the ensemble have a mean mass flux $\langle m\rangle$ that we take to be a fixed constant. Note that a conventional mass-flux parameterization requires only $\langle M\rangle$ and is insensitive to $\langle m\rangle$. In the stochastic context, however, $\langle m\rangle$ (or $1 /\langle N\rangle)$ sets the scale for the fluctuations of $M$ about $\langle M\rangle$. Although the available information about $\langle m\rangle$ is limited, there are indications that a constant value provides a reasonable first approximation. It is found in CRM studies (Robe and Emanuel 1996; Shutts and Gray 1999; Cohen 2001) that the strength of an imposed forcing has only a weak effect on the mean vertical velocity of individual updrafts, consistent with the scalings of Emanuel and Bister (1996) and Grant and Brown (1999). Thus, an increase to the forcing is associated predominantly with an increase to the fractional area of updrafts. Cohen (2001) has gone further by suggesting that changes to the fractional area may be largely attributable to a change in the number of updrafts, with changes to their size being a subsidiary effect. Figure 3 supports this contention by showing CRM results for $\langle m\rangle$ as a function of height for different strengths of forcing. A doubling of the imposed tropospheric cooling increases the total mass flux by a similar factor (Cohen 2001; see also Robe and Emanuel 1996, their Fig. 7a) but leaves the mean mass flux per updraft essentially unchanged. Interestingly, the figure also reveals that there is only a weak dependence of $\langle m\rangle$ on height in the midtroposphere, suggesting that the midlevel changes in total mass flux with height in these simulations are associated predominantly with changes to the number of clouds reaching each level. Based on Fig. 3, we have chosen $\langle m\rangle=2 \times 10^{7} \mathrm{~kg} \mathrm{~s}^{-1}$ for the initial tests of the stochastic parameterization.

The variability associated with different numbers of clouds appearing in a subregion will depend on the de- 
(a)

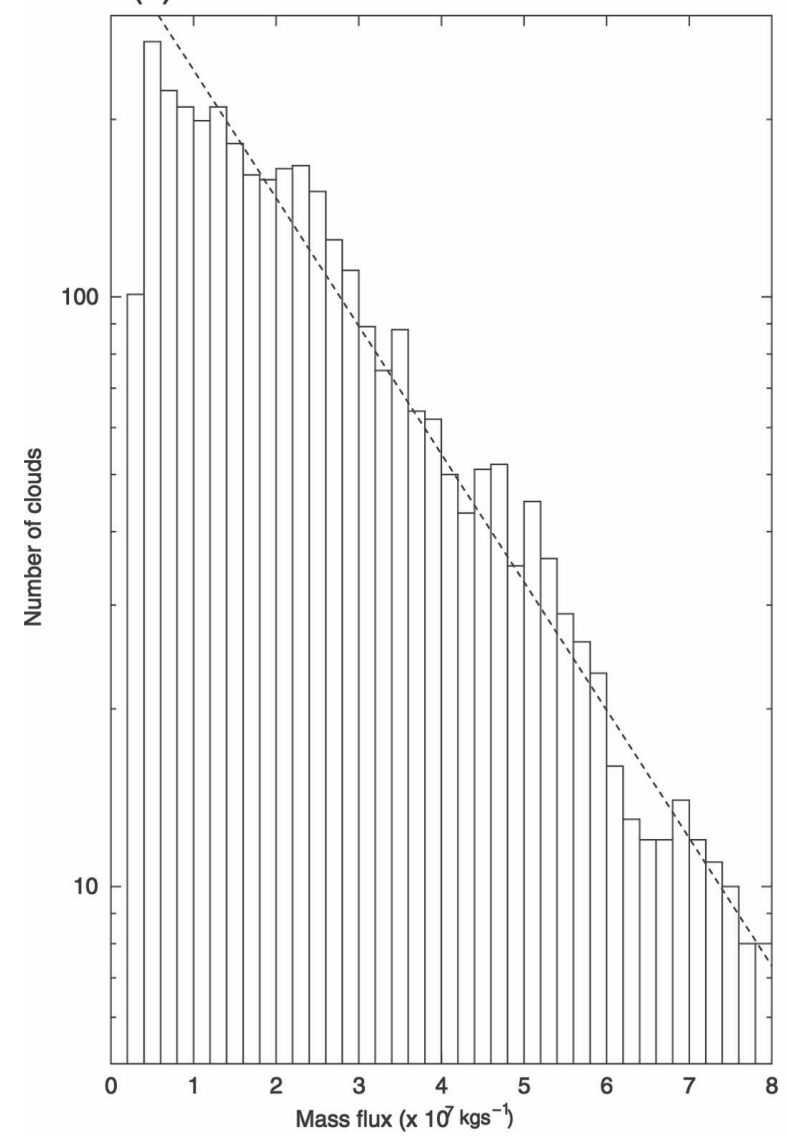

(b)

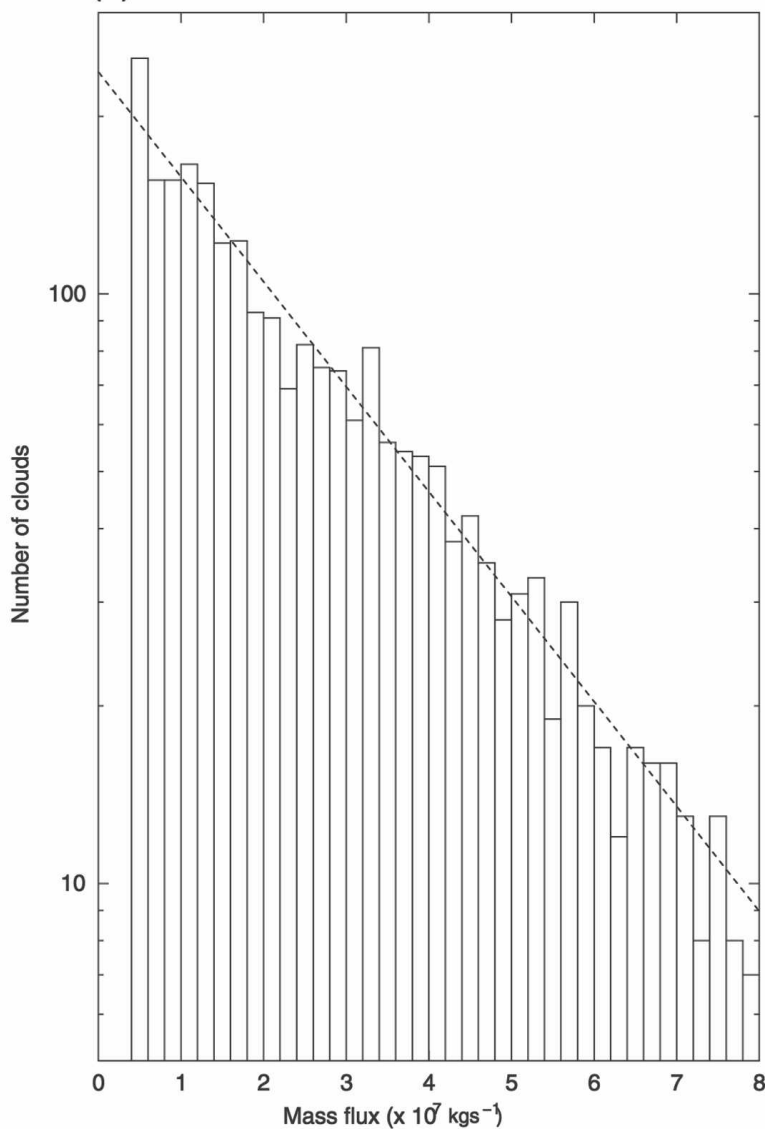

FIG. 2. Histograms of updraft mass fluxes obtained from CRM simulations (Cohen 2001) of radiative-convective equilibrium. The mass flux is binned into intervals of $2 \times 10^{6} \mathrm{~kg} \mathrm{~s}^{-1}$ and lines of best fit for an exponential distribution have been added for a cooling rate of (a) $8 \mathrm{~K} \mathrm{day}^{-1}$ at $3.1 \mathrm{~km}$ and (b) $16 \mathrm{~K} \mathrm{day}^{-1}$ at $1.3 \mathrm{~km}$. Data are taken from the equilibrium state using (a) 98 times over 4 days and (b) 30 times over 1.3 days.

gree of spatial organization of the convection. The simplest assumption is that the clouds are randomly distributed in space, and this was found to be a reasonably accurate approximation, even in simulations where a strong environmental shear was imposed, leading to squall line-like organization (Cohen and Craig 2006). In such cases, the standard deviation of the mass flux in a given region was within about $10 \%$ of the value for a completely random spatial distribution. Craig and Cohen (2006) have shown that an exponential distribution for the mass flux of each cloud then implies a PDF for the total mass flux in a region given by

$$
\begin{aligned}
p(M)= & \frac{1}{\langle m\rangle} \sqrt{\frac{\langle M\rangle}{M}} \exp \left(-\frac{M+\langle M\rangle}{\langle m\rangle}\right) \\
& \times I_{1}\left(\frac{2}{\langle m\rangle} \sqrt{\langle M\rangle M}\right),
\end{aligned}
$$

where $I_{1}$ denotes the modified Bessel function of order 1 .

\section{b. Outline of the stochastic parameterization}

The stochastic parameterization is based on the equilibrium distribution described above. The convection in a grid box will be described by the number of clouds of each mass flux present at a given time. Individual clouds are assumed to have a size much smaller than a grid box and are distributed randomly in space, leading to no correlation between the mass flux occurring in adjacent grid boxes. On the other hand, clouds may have a finite lifetime, and at each time step new clouds are initiated by choosing randomly from the distribution of Craig and Cohen (2006).

The distribution of Craig and Cohen (2006) has two parameters, $\langle M\rangle$ and $\langle m\rangle$. The ensemble-mean total cloud-base mass flux, $\langle M\rangle$, comes from a closure assumption, in common with other mass-flux-based parameterizations, while the mean cloud-base mass flux of an individual cloud, $\langle m\rangle$, is assumed constant as discussed above. Another aspect in common with conven- 


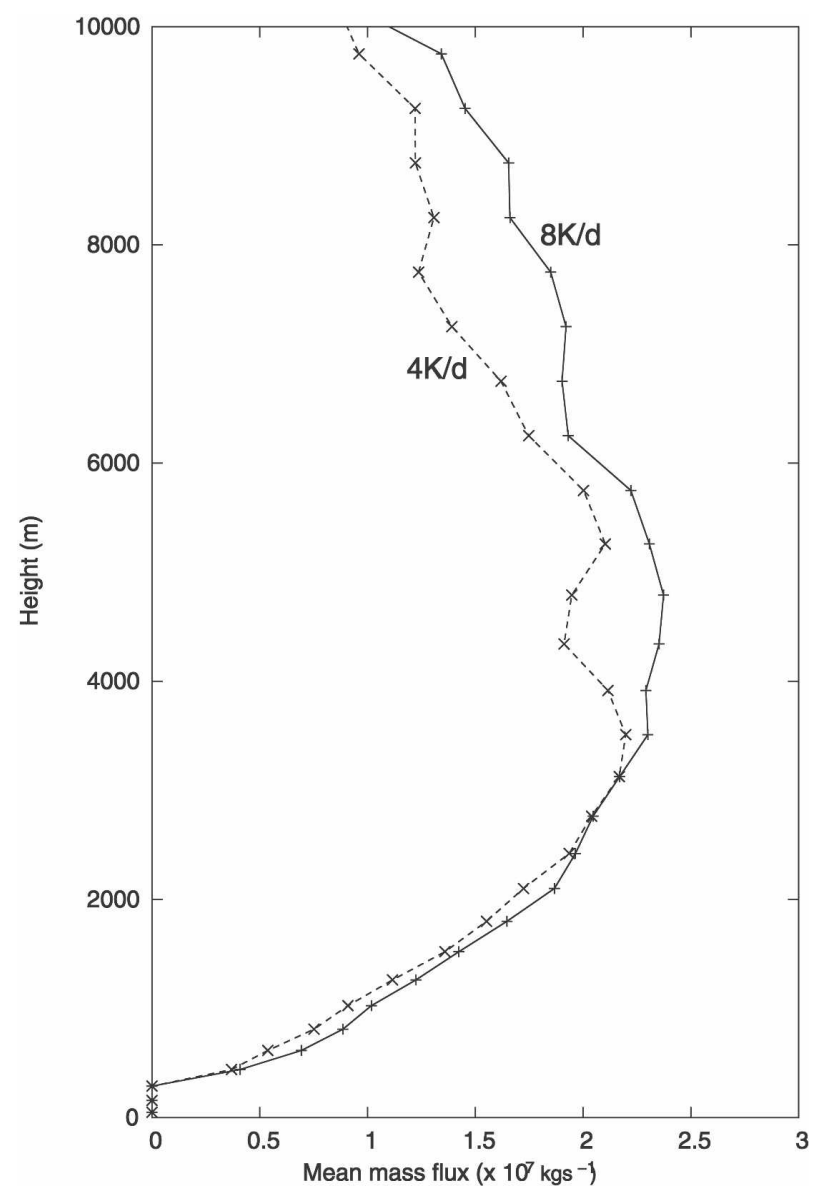

FIG. 3. The mean mass flux of a convective updraft obtained from CRM simulations of radiative-convective equilibrium (Cohen and Craig 2006). The mean is calculated for each vertical level, using 98 times over 4 days (for the $8 \mathrm{~K} \mathrm{day}^{-1}$ cooling rate) and 159 times over 7.75 days (for the $4 \mathrm{~K} \mathrm{day}^{-1}$ cooling rate).

tional mass-flux parameterizations is the computation of vertical profiles of temperature and moisture tendencies from the cloud-base mass flux, using a cloud model such as an entraining plume (Arakawa and Schubert 1974; Ooyama 1971).

With these assumptions, the four steps of the general algorithm presented earlier can be restated more precisely:

1) Compute large-scale properties by horizontally averaging vertical profiles of temperature and moisture over a region centered on each grid point. The size of the region is proportional to the length scale $L=\sqrt{\langle m\rangle / \overline{\langle M\rangle}}$, where $\overline{\langle M\rangle}$ is the ensemble-mean total cloud-base mass flux per unit area. Here $L$ is a measure of the separation between clouds. In principle this scale is variable, depending on the output of the closure calculation, and iteration is required to define an averaging region consistent with the resulting $\overline{\langle M\rangle}$. As discussed later, no such iteration is implemented for the single-column tests in this paper.

2) Compute equilibrium convective distributions, with $\langle M\rangle$ given by the closure applied to the spatially averaged sounding and $\langle m\rangle$ assumed constant. Along with Eq. (1), these parameters give the distribution of the number of clouds of each cloud-base mass flux in a grid box of a given size.

3) Given the lifetime of a cloud and the time step, the probability of initiation of clouds of each cloud-base mass flux in the grid box can now be computed. Clouds at the end of their lifetime are removed and new clouds are initiated randomly according to the specified distribution. Note that if the size of the grid box is large (compared with the averaging length from step 1), each grid box will contain a representative sample of the entire distribution of cloud sizes, and the parameterization will converge to a deterministic equilibrium mass-flux scheme with a spectral cloud model.

4) Compute large-scale convective tendencies based on the population of clouds in the grid box. A cloud model is used to compute vertical profiles of tendencies for each cloud based on its cloud-base mass flux.

To this point we have not specified which closure assumption will be used to compute $\langle M\rangle$ (step 2), nor which cloud model will be used for the tendency profiles (step 4). Many choices are possible, but for the present work, these elements will be based on the Kain-Fritsch parameterization scheme (KF; Kain and Fritsch 1990, 1993; Kain et al. 2003; Kain 2004). This is a state-of-the-art cumulus parameterization designed for mesoscale models and widely used in research and operational forecasting. A brief description of the KF scheme can be found in the appendix. Using this existing scheme as a basis has the advantage that it is robust and well-tested code, and allows the original KF scheme to be used as a reference point for testing.

The cloud model is an entraining/detraining plume with relatively detailed microphysics. Some details of the KF plume model have been adapted for the present purposes, notably the determination of cloud-base properties for each plume, and the cloud lifetime, as described in section 3 . The vertical profiles of temperature and moisture tendency are calculated as sums over the population of plumes in the grid box. The closure assumption is to set $\langle M\rangle$ to be sufficient to remove CAPE over a specified time scale. The closure time scale will depend on forcing via the cloud spacing, fol- 
lowing Cohen and Craig (2004), and discussed in section 4.

\section{The cloud model}

\section{a. The ensemble of plumes}

The plume model from the KF scheme is used to specify the behavior of each cloud in the distribution. Plumes are required with a full range of mass fluxes at the lifting condensation level (LCL) in order to create an exponential distribution [Eq. (1)] there. Exponential distributions should also occur at higher levels (section 2a) but are not imposed by the parameterization; in section $5 b$, we test whether the plume ensemble is capable of maintaining exponential distributions aloft. In this section, we consider how a desired cloud-base mass flux is assigned to a plume.

In the plume model, the maximum entrainment rate for the updraft is inversely proportional to updraft radius. In the KF parameterization a single radius is used to represent all convection within a grid box. Here, a spectrum of clouds can be obtained by allowing a spectrum of entrainment rates (radii). Although the values used for the updraft radii should not be interpreted too literally (Emanuel 1994; Kain 2004) it may nonetheless be reasonable to assume that the radius-like parameter in the entrainment formulation provides some meaningful measure of updraft size. Indeed, Kuang and Bretherton (2006) have recently found support for this notion from CRM simulations. We will assume the relationship

$$
m=\frac{\langle m\rangle}{\left\langle r^{2}\right\rangle} r^{2}
$$

The equation essentially requires that the vertical velocities in updrafts be independent of updraft size (and hence of the entrainment rate). Such a condition is manifestly false within the body of an updraft but may hold close to the level where it is initiated. Eqs. (1) and (3) are used at the LCL to provide a PDF of plume radii, or equivalently of entrainment rates. We remark that the ensemble scheme of Frank and Cohen (1985) also relies on a transformation between cloud size and mass-flux distributions, which is achieved by an equivalent relationship. A similar assumption was made by Donner (1993).

The probability in a single time step (of duration $d t$ ) of initiating a plume of radius $r$ in the range $r$ to $r+d r$ with a cloud-base mass flux given by Eq. (3) can now be written as

$$
\langle N\rangle p(r) d r \frac{d t}{T}=\frac{\langle M\rangle}{\langle m\rangle} \frac{2 r}{\left\langle r^{2}\right\rangle} \exp \left(\frac{-r^{2}}{\left\langle r^{2}\right\rangle}\right) d r \frac{d t}{T},
$$

where $T$ is the lifetime for which a plume persists. In principle, $T$ may be a function of plume radius and properties of the large-scale environment. ${ }^{1}$ Here, we make the simple choice ${ }^{2}$ of $T=45 \mathrm{~min}$.

A description of the closure for determining $\langle M\rangle$ is deferred to section 4. Assuming this to be known, random numbers in the range 0 to 1 can then be generated ${ }^{3}$ for each possible updraft radius and tested against the probabilities given by Eq. (4). The probability of initiation has a maximum for $r=\sqrt{\left\langle r^{2}\right\rangle / 2}$, and the radius interval $d r$ is selected such that this probability is $5 \%$, subject to a maximum bin width of $50 \mathrm{~m}$.

The convective clouds at a given moment consist of plumes initiated at the current time step, together with plumes initiated previously whose lifetimes have not expired. Thus, a preexisting set of plumes should be specified as part of the initial conditions for a model run. If such information (from a previous model run) is not available, it is convenient to generate a full set of plumes during the first time step. This can be achieved by neglecting the factor $d t / T$ in Eq. (4). Plumes generated in this fashion are not to be designated as newly initiated clouds but have ages assigned randomly as a uniform distribution extending up to the full lifetime.

\section{b. Adaptations of KF plume model}

When a plume is initiated its characteristics above cloud base are unconstrained by the theory of section 2 . They could be regarded as depending on the local (grid box) or on the large-scale environmental state. (In the latter case, the whole parameterization would then depend only on large-scale properties.) Both possibilities have been explored (section 5d). Regardless of whether the input sounding is local or spatially averaged, the KF plume model is used to calculate plume characteristics aloft, albeit with some adaptations that are desirable for our present purposes. This section outlines modifications to the choice of source layer and initial temperature perturbation, and notes how negative moisture tendencies are treated.

The plume model considers a sequence of potential updraft source layers, each spanning a whole number of

\footnotetext{
${ }^{1}$ Within an ensemble of clouds, one might expect a larger cloud to persist longer than a smaller one. It would certainly be interesting to collect some quantitative information on this point, perhaps by introducing an element of cloud tracking to CRM simulations.

${ }^{2}$ Other choices of lifetime produced only minor changes to the radiative-convective equilibrium state described in section 5 .

${ }^{3}$ The random number generator is the minimal Park and Miller method with Bays-Durham shuffle and added safeguards, as presented in Press et al. (1992).
} 
model levels. This proved problematic in that changes to the identity of the actual updraft source layer between time steps could result in jumps in the height of the LCL that are undesirable if attempting to close the parameterization there. We achieve smoother variations in the LCL by defining potential source layers to be exactly $50 \mathrm{mb}$ deep, the base of each being $5 \mathrm{mb}$ higher than the previous potential layer tested. Note that once a suitable source layer has been found it is then held fixed for a given sounding: that is, the source layer is not permitted to vary with plume radius.

Another issue related to the search for a suitable updraft source layer is the temperature perturbation applied when testing for buoyancy at the LCL. This has been simplified to use a fixed perturbation of $0.2 \mathrm{~K}$, similarly to the Gregory and Rowntree (1990) scheme. However, should no buoyant source layer be identified then the search is repeated with the perturbation incremented in steps of $0.1 \mathrm{~K}$. When a parcel is released at the LCL, the updraft calculations assume an initial velocity dependent upon the temperature perturbation. In practice, we have found that, for any perturbation beyond threshold, reasonable variations in its strength have little consequence. This agrees with Nober's (2003) observation for a different plume model.

Each convective plume present has associated tendencies of the grid box moisture variables (in our case water vapor, cloud water, and cloud ice). With multiple plumes present over multiple time steps, there is a possibility of producing a negative value for one of the moisture variables. In such an eventuality, the offending variable is simply reset to zero by borrowing moisture of another type (including a corresponding latent heat adjustment). This procedure may be insufficient if a required drying exceeds the total moisture available, and in that case all plumes present that have a drying tendency on the problematic model level are removed before the end of their envisaged lifetime. Although occasional small changes of this type are necessary, it is reassuring to note that removal of plumes is extremely rare in our experience.

\section{Implementation of the CAPE closure}

Calculation of the required ensemble-mean mass flux $\langle M\rangle$ is based on the deep-convection closure of the KF parameterization (see appendix), extended to apply to an ensemble of plumes. The plume spectrum is divided into 50 equal radius intervals ranging up to $2 \mathrm{~km}$, with each spectral element described by the entraining/ detraining plume model. The ensemble-mean mass-flux profile and dilute CAPE are determined from the sum over spectral elements, weighted by their probability of occurrence [Eq. (1)]. Mass fluxes are scaled ${ }^{4}$ such that at least $90 \%$ of the dilute CAPE would be removed were the full ensemble of plumes to act on the largescale environmental sounding for a time $T_{c}$, the closure time scale. The extended closure has been validated by a number of explicit checks: for example, taking an "ensemble" of plumes all having the same updraft radius and with arbitrary weightings that sum to unity, the method produces identical results to the KF closure using that radius.

The closure time scale can be interpreted as the adjustment time in response to a change of forcing (if the forcing were removed, convection would decay in this time). Following Cohen and Craig (2004), we relate this time scale to the cloud separation in the large-scale environment, such that

$$
T_{c}=k L=k \sqrt{\frac{\langle m\rangle}{\langle\bar{M}\rangle}},
$$

where $k$ is a constant, which will depend on the definition of adjustment. This relationship is consistent with the view that the response to a change of forcing is governed by the time taken for a gravity wave signal to propagate between clouds (Bretherton and Smolarkiewicz 1989) since the average speed of convectively generated gravity waves appears to be rather insensitive to the large-scale environment (Cohen and Craig 2004). Equation (5) produces a closure time scale that is relatively long with weak forcing but short with strong forcing.

\section{Tests of the scheme}

In this section, we test the behavior of the stochastic scheme. A precondition before more ambitious use of the scheme is that it should be capable of replicating both the mean convective state and the statistical fluctuations about that state for the situation in which Eqs. (1) and (2) are known to hold. To test this, we perform single-column model (SCM) experiments that aim to replicate the radiative-convective equilibrium CRM simulations of Cohen and Craig (2006). In particular, we address the following questions:

1) For a steady external forcing, does the convective variability become small with increasing grid box size? (Section 5b.)

\footnotetext{
${ }^{4}$ Subject to the constraint of a maximum scaling governed by the mass in each layer. There are also some simplifications for extremely weak forcings and facilities for dealing with numerical problems in the iteration procedure. These are broadly similar to those in the KF code.
} 
2) Are the time-mean temperature and humidity profiles reasonable (comparable with those obtained using the unmodified KF scheme)? (Section 5b.)

3) Are the properties of individual plumes consistent with CRM results (the exponential distribution of plume mass fluxes and the approximate constancy of ensemble-mean mass flux with height)? (Section 5c.)

4) Does the variability in a finite-sized grid box follow the prescribed distribution of $M$ [Eq. (2)]? (Section $5 d$.)

5) Is the parameterization stable under steady external forcing (can the imposed variability be removed by time averaging to provide stable input, representative of the large-scale environment, for the closure calculation in subsequent time steps)? (See section $5 d$.)

\section{a. SCM arrangement}

The single-column configuration of the Unified Model (UM; Cullen 1993) is used. Apart from the radiation (imposed) and convection (under test) schemes, we employ the physical parameterizations available in version 4.5 of that model. Layer clouds are parameterized using the Smith (1990) scheme with associated precipitation represented as in Smith et al. (1998) and Wilson and Ballard (1999). The variables considered are for water vapor, liquid water, frozen water, and rain. Total cloud condensate is determined by assuming a triangular probability distribution function of cloudconserved variables about the grid box mean (Smith 1990). Various microphysical processes (Wilson and Ballard 1999) make transfers between the moisture variables, with frozen water being treated prognostically while liquid water and rain are diagnostic.

Surface fluxes are represented as simple linear functions of the temperature and moisture differences between the first model level and the surface. Constants of proportionality are derived from the mean fluxes that occur in the equilibrium state of the corresponding CRM run (B. Cohen 2003, personal communication). In the absence of any boundary layer shear in these SCM experiments, the boundary layer mixing of temperature, water vapor, and layer-cloud water (Smith 1990) is calculated with a first-order turbulence closure and using free-convective scalings for the eddy diffusivities (Smith and Williams 2000).

Sea surface temperature is held fixed at $300 \mathrm{~K}$, and 49 sigma surfaces are chosen to correspond approximately with the vertical resolution of the CRM simulations.

As in the CRM simulations, explicit radiation calculations are replaced by a prescribed tropospheric cooling rate, which is constant up to $400 \mathrm{mb}$ and decays to
TABLE 1. Default parameter choices for the stochastic convective parameterization, as used in the SCM runs of section 5. In each case the choice made is discussed in the section referred to in the final column. Here $N_{s}$ denotes the number of soundings that are time averaged to provide large-scale profiles. All other symbols are defined in the main text.

\begin{tabular}{lcc}
\hline \hline Parameter & Value & Reference section \\
\hline$\langle m\rangle$ & $2 \times 10^{7} \mathrm{~kg} \mathrm{~s}^{-1}$ & $2 \mathrm{a}$ \\
$T$ & $45 \mathrm{~min}$ & $3 \mathrm{a}$ \\
$\sqrt{\left\langle r^{2}\right\rangle}$ & $450 \mathrm{~m}$ & $5 \mathrm{~b}$ \\
$k$ & $0.3 \mathrm{~s} \mathrm{~m}^{-1}$ & $5 \mathrm{~d}$ \\
$N_{s}$ & 90 & $5 \mathrm{~d}$ \\
\hline
\end{tabular}

zero linearly with pressure up to $200 \mathrm{mb}$. The cooling rate is here set at $8 \mathrm{~K} \mathrm{day}^{-1}$. Budget diagnosis of preliminary runs showed that the only process operating above $200 \mathrm{mb}$ was the convection scheme, which occasionally produced cooling at the top of the deepest clouds. The result was a drift in potential temperature around the tropopause. This is an artifact of the simple prescribed radiative cooling; similar changes also occur in CRM simulations (B. Cohen 2003, personal communication; C. Roadnight 2001, personal communication). We therefore introduced a Newtonian relaxation of the potential temperature toward its initial state above 200 $\mathrm{mb}$ with a relaxation time of a few hours.

Unless otherwise stated, the SCM uses the default set of parameters listed in Table 1 . The table includes references to section numbers where discussion can be found on the choice of each parameter and relevant sensitivities. It should be noted that, in the results to be presented here, calculations of initiated plumes were based on instantaneous soundings (see section $3 \mathrm{~b}$ ). The alternative of using a time-averaged sounding to represent the large-scale environment produced almost identical results for these single-column tests.

It is convenient in the SCM to use a time step of 5 min so that there are several time steps within the specified cloud lifetime $T$.

An SCM equilibrium state was also obtained with the unmodified KF parameterization for comparison purposes. This required an additional assumption since there was no column-scale vertical velocity available for calculation of a temperature perturbation, $\delta T$ (see appendix). Instead, $\delta T$ was taken to be constant. There is some sensitivity to the choice made. While a deep plume is present approximately $40 \%$ of the time, shallow plumes can be relatively rare (small $\delta T$ ) or prevalent $(\delta T \geqslant 0.1 \mathrm{~K})$. For $\delta T \geqslant 0.2 \mathrm{~K}$, the boundary layer structure starts to suffer, becoming unrealistically shallow. Reasonable agreement with mean thermodynamic profiles from the CRM was obtained for $0.02 \lesssim \delta T \lesssim$ $0.2 \mathrm{~K}$, with shallow convection occurring more than 
$30 \%$ of the time. Results presented in this paper are for $\delta T=0.05 \mathrm{~K}$.

\section{b. Tests of mean state}

We consider first a column that represents a large "grid box" of side $400 \mathrm{~km}$. Some statistics at equilibrium with the default parameter set are given in Table 2. It is immediately apparent that the SCM produces fewer clouds per unit area than the CRM. We shall see in section $5 \mathrm{c}$ that, while there are considerably fewer shallow clouds in the SCM, the numbers penetrating to the midtroposphere are similar. The SCM column contains $\sim 180$ clouds present at any instant. The stochastic aspect of the parameterization is therefore weak and the variability of convective properties is small (see Table 2). This is convenient for testing the equilibrium state.

With the steady external forcing used in these SCM experiments, the total mass-flux response $M$ at equilibrium for a large grid box should also be steady. Figure 4 shows normalized time series of mass flux. The convective response is, indeed, steady when running the SCM over large areas (Figs. 4a,b). By contrast, significant time variations occur when using the KF parameterization (Fig. 4d). Such variations are purely artificial: the results of a conventional mass-flux parameterization are independent of grid box area but sensitive to details of the triggering (as noted above, on-off behavior occurs when using the KF scheme with this forcing for small $\delta T)$. Coincidentally, a similar level of variability is produced by the stochastic parameterization operating over an area of $(64 \mathrm{~km})^{2}$ (Fig. $\left.4 \mathrm{c}\right)$. In section $5 \mathrm{~d}$ we check that these variations are appropriate by investigating the PDF of total mass flux.

Figure 5 shows thermodynamic profiles from the equilibrium state of the CRM, along with the departures from that state that occur in various SCM experiments. The equilibrium states from the SCM are somewhat moister within the boundary layer and lower troposphere and somewhat drier and cooler above. Departures of this size, however, are consistent with expectations for a change of modeling framework: the corresponding radiative-convective equilibrium profiles obtained from a different CRM (C. Roadnight 2001, personal communication) exhibit differences of similar size to those between the SCM and CRM states. $^{5}$

The time-averaged equilibrium state in the SCM is not significantly modified by the larger fluctuations that

\footnotetext{
${ }^{5}$ See also, for example, Guichard et al. (2004) for a comparison of CRMs and SCMs in simulations of the diurnal cycle of deep convection.
}

TABLE 2. Statistics of the SCM equilibrium state produced by the stochastic convective parameterization. The SCM represented an area $(400 \mathrm{~km})^{2}$. "SCM mean" values are calculated as time averages between the 10th and 20th days. "Dilute" CAPE is that calculated for the plume ensemble (section 4). Also given (where appropriate) are corresponding values obtained from the domainaveraged state of the companion CRM experiment. This was run over a domain area $(128 \mathrm{~km})^{2}$, so the number of clouds has been scaled up by the ratio of SCM to CRM areas to provide an appropriate comparison.

\begin{tabular}{lccc}
\hline \hline \multicolumn{1}{c}{ Quantity } & $\begin{array}{c}\text { Mean } \\
\text { SCM }\end{array}$ & $\begin{array}{c}\text { Standard } \\
\text { deviation }\end{array}$ & $\begin{array}{c}\text { Mean } \\
\text { CRM }\end{array}$ \\
\hline Closure time scale $T_{c}(\mathrm{~min})$ & 144 & 6 & - \\
LCL $(\mathrm{m})$ & 1297 & 72 & 1839 \\
Number of clouds $\langle N\rangle$ & 181 & 19 & 449 \\
Forcing at LCL $\overline{\langle M\rangle}$ & 0.0243 & 0.0018 & 0.0634 \\
$\quad\left(\mathrm{~kg} \mathrm{~m}^{-2} \mathrm{~s}^{-1}\right)$ & & & \\
Dilute CAPE $\left(\mathrm{J} \mathrm{kg}^{-1}\right)$ & 212 & 36 & - \\
Surface heat flux $\left(\mathrm{W} \mathrm{m}{ }^{-2}\right)$ & 102 & 2 & 102 \\
Surface latent heat flux & 546 & 8 & 557 \\
$\quad\left(\mathrm{~W} \mathrm{~m}{ }^{-2}\right)$ & & & \\
\hline
\end{tabular}

occur at individual time steps when running over smaller areas. For model levels below $12 \mathrm{~km}$, the rms differences between equilibrium states when running over areas of $(400 \mathrm{~km})^{2}$ and $(64 \mathrm{~km})^{2}$ are $0.23 \mathrm{~K}$ and $0.11 \mathrm{~g} \mathrm{~kg}^{-1}$ for potential temperature and water vapor, respectively. In comparison, the rms differences in states between $\mathrm{SCM}$ runs at $(400 \mathrm{~km})^{2}$ using rms radii of 450 and $600 \mathrm{~m}$ (as in Fig. 5) are $0.49 \mathrm{~K}$ and $0.31 \mathrm{~g}$ $\mathrm{kg}^{-1}$.

The stochastic parameterization contains a rms radius parameter $\sqrt{\left\langle r^{2}\right\rangle}$ that governs the proportions of small and large plumes. Preliminary tests suggested that a good balance is obtained for $\sqrt{\left\langle r^{2}\right\rangle} \sim 450 \mathrm{~m}$, and we have adopted this as a default value (Table 1). Results for other choices of the parameter are shown in Figs. 5 and 6 . The changes are modest. With an increased weighting for small clouds, it is necessary for the plumes to be more penetrative: this is achieved by weakening the inversion, with a cooler mid-to-upper troposphere and a slightly warmer boundary layer. A consequence is that the lower-tropospheric peak in the updraft massflux profile is raised for a smaller rms radius (Fig. 6). Otherwise the mass-flux profile is rather insensitive to the rms radius, and effects on the heating budget are likewise minor (not shown).

\section{c. Tests of individual plume properties}

Let us now consider the behavior of individual plumes within the SCM equilibrium state. Running the plume model offline for various updraft radii produces the mass-flux profiles shown in Fig. 7. The profiles are highly sensitive to radius, as recognized by Kain and 
(a)

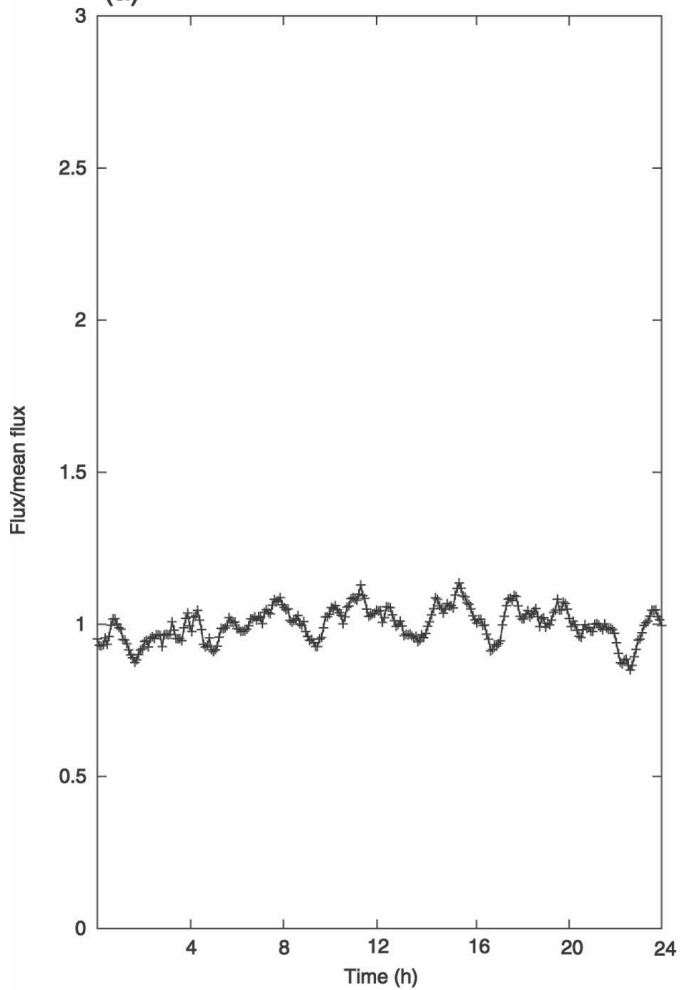

(c)

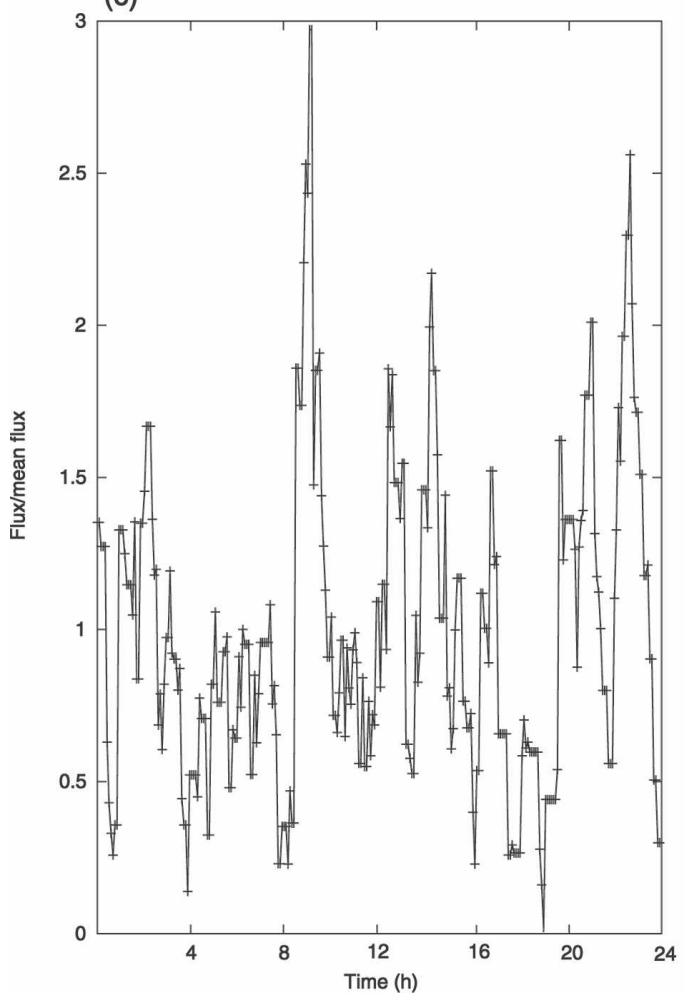

(b)

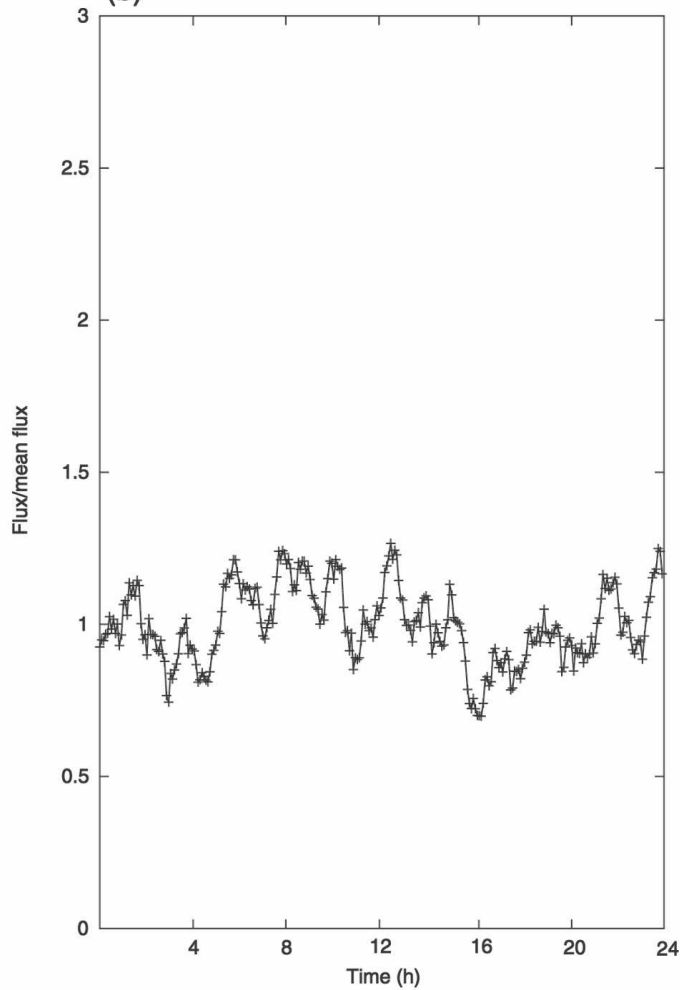

(d)

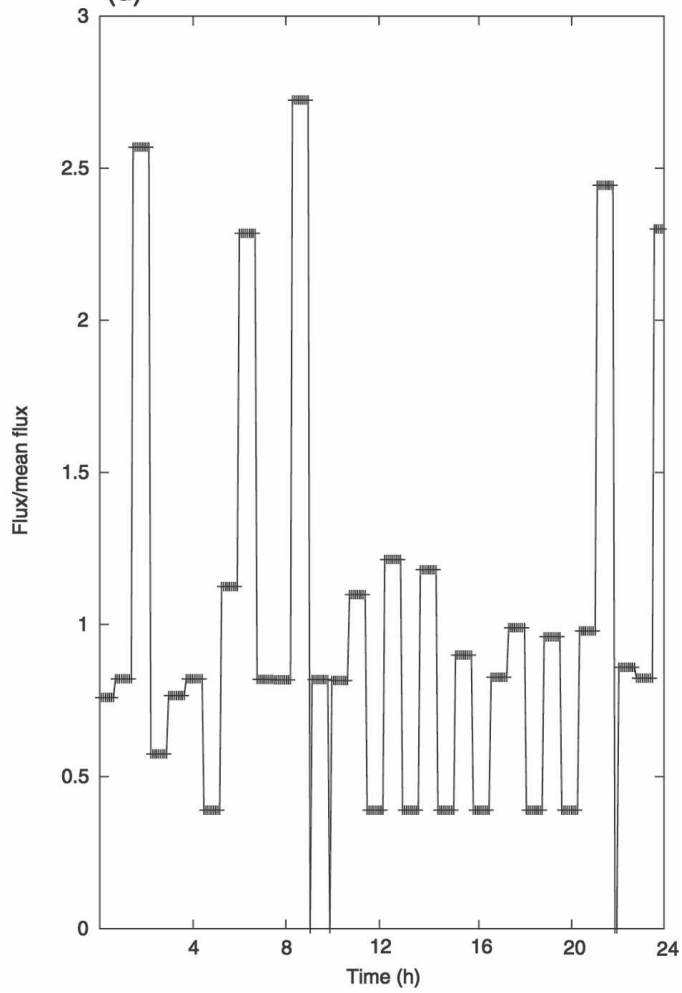

FIG. 4. Time series of the total mass flux $M$ at $1.8 \mathrm{~km}$ for the 10 th day of various SCM experiments. The mass flux has been normalized by its time-mean value for that day. The SCM runs are for the stochastic parameterization over areas of (a) $(1000 \mathrm{~km})^{2}$, (b) $(400 \mathrm{~km})^{2}$, and (c) $(64 \mathrm{~km})^{2}$, and for (d) the KF parameterization (area irrelevant). 
(a)

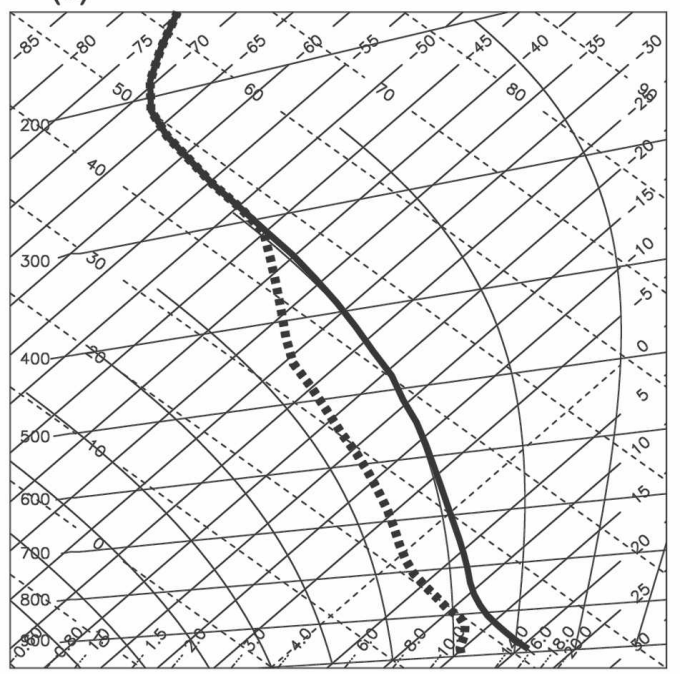

(b)

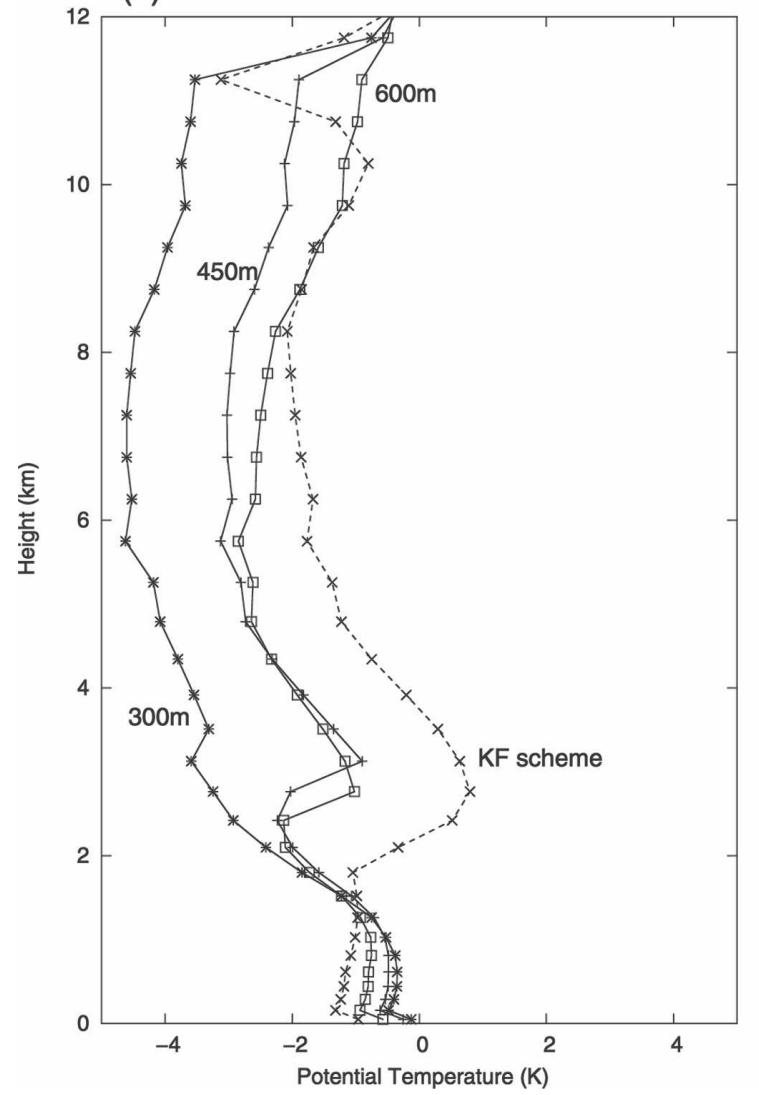

(c)

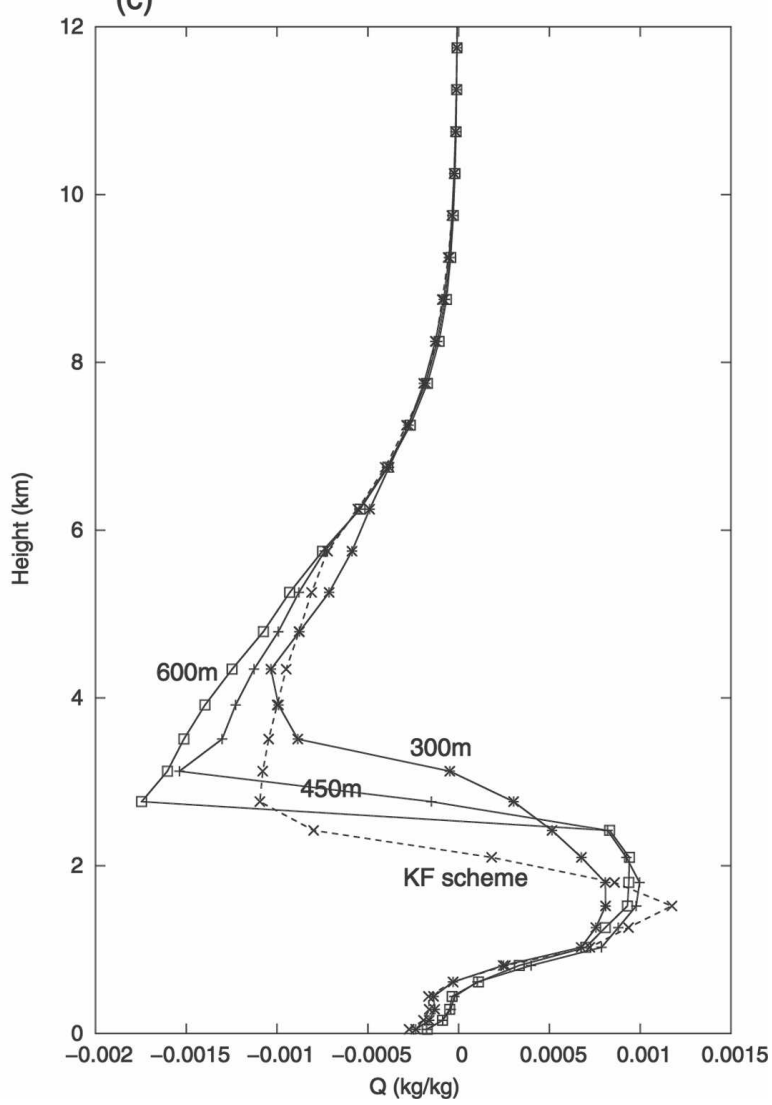

FIG. 5. (a) Tephigram showing the spatially averaged temperature (solid, bold line) and dewpoint (dotted, bold line) from the CRM equilibrium state. Also shown are the time-averaged departures from that state for (b) the potential temperature and (c) the water vapor obtained at equilibrium in various SCM experiments. The SCM runs use either the stochastic parameterization (solid, with different values for $\sqrt{\left\langle r^{2}\right\rangle}$ marked), or the KF parameterization (dashed). Results from the SCM have been averaged over the 10th day of each run. 


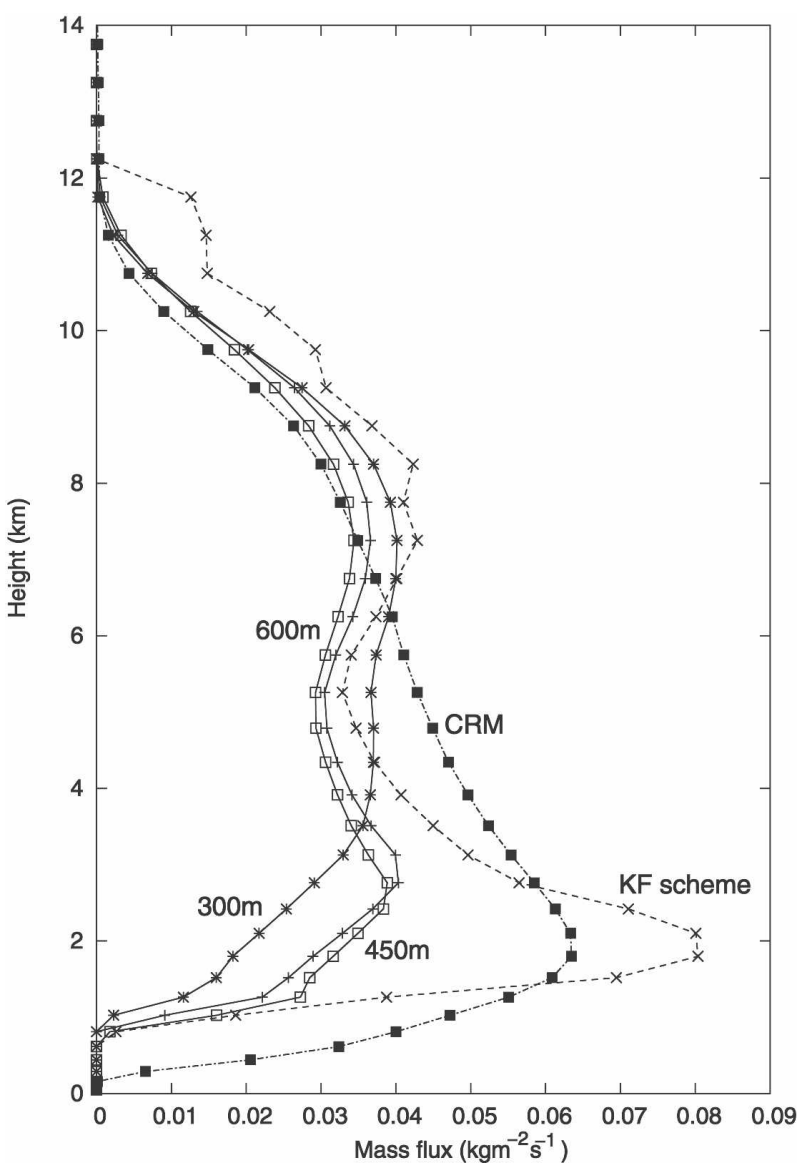

FIG. 6. Profiles of the total convective mass flux per unit area obtained at equilibrium from the CRM (Cohen 2001) and from various SCM experiments. The SCM runs use either the stochastic parameterization (solid, with different values for $\sqrt{\left\langle r^{2}\right\rangle}$ marked) or the KF parameterization (dashed). Results from the SCM have been averaged over the 10th day of each run.

Fritsch (1993). The unmodified KF parameterization uses a radius of $1 \mathrm{~km}$ and this produces a deepconvective profile that is in marked contrast ${ }^{6}$ to that found in the CRM (Fig. 6). A reasonable balance between deep and shallow convection can be achieved when using the KF parameterization in the SCM (Fig. 6), but only by generating artificial variations in time between deep, shallow, and no convection (Fig. 4b). Allowing a spectrum of plumes leads to a weighted sum over cloud types without requiring artificial fluctuations of a model sounding.

Plumes that entrain very strongly (i.e., with small up-

\footnotetext{
${ }^{6}$ Although mass fluxes from a CRM and a convective parameterization are not directly comparable, gross deviations in the basic shape of the profiles can nonetheless be regarded as significant.
}

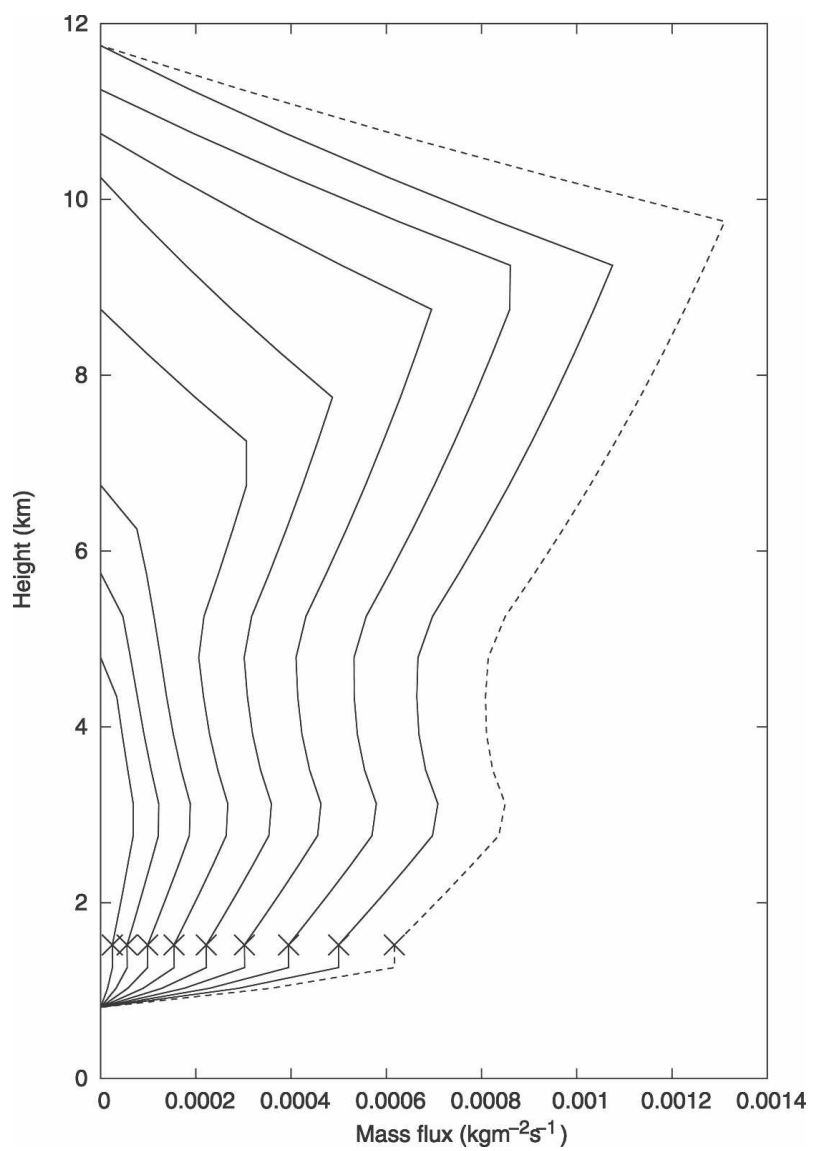

FIG. 7. Profiles of updraft mass flux per unit area produced by running the plume model offline and using the time-averaged SCM equilibrium state from the run over an area of $(400 \mathrm{~km})^{2}$. Each profile corresponds to a particular updraft radius. Following the profiles from left to right across the figure corresponds to an increase in radius from $200 \mathrm{~m}$ to $1 \mathrm{~km}$, in steps of $100 \mathrm{~m}$. A profile corresponding to the KF parameterization is indicated by a dotted line. The profiles are normalized by applying Eq. (3) at the LCL, the normalization points indicated with a cross.

draft radii) are unable to rise even a single model level. These are simply ignored by the stochastic parameterization. For the SCM runs discussed here, the spectral truncation typically results in losses of $\sim 1 \%$ of the total mass flux and $\sim 3 \%$ of the cloud number. Experiments with several "correction" methods to restore the missing mass flux and cloud number confirm that the truncation has no significant effect for any of the tests in this paper.

The distributions of plume mass fluxes in the SCM equilibrium state provide an important test of the physical basis for our parameterization. Recall from section $2 \mathrm{a}$ that theory predicts an exponential distribution for any fixed height in the atmosphere. Although an exponential distribution is imposed at the LCL, other distributions may be established at other levels. It 
(a)

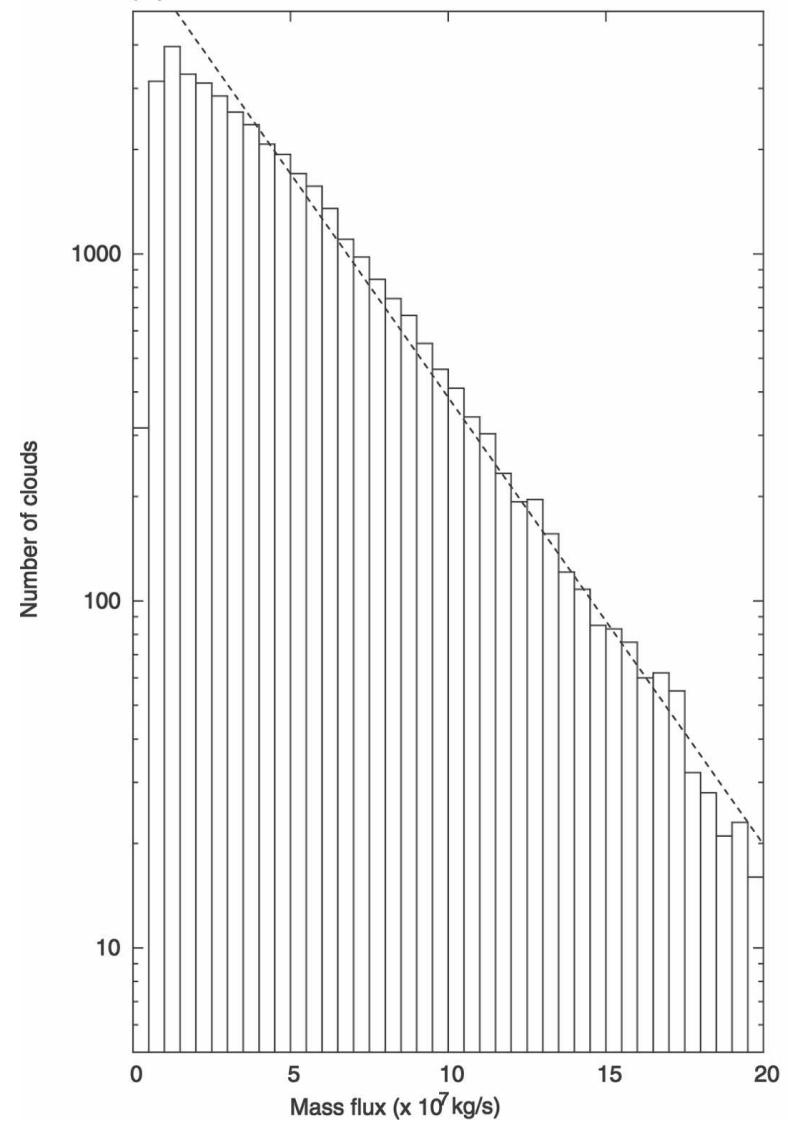

(b)

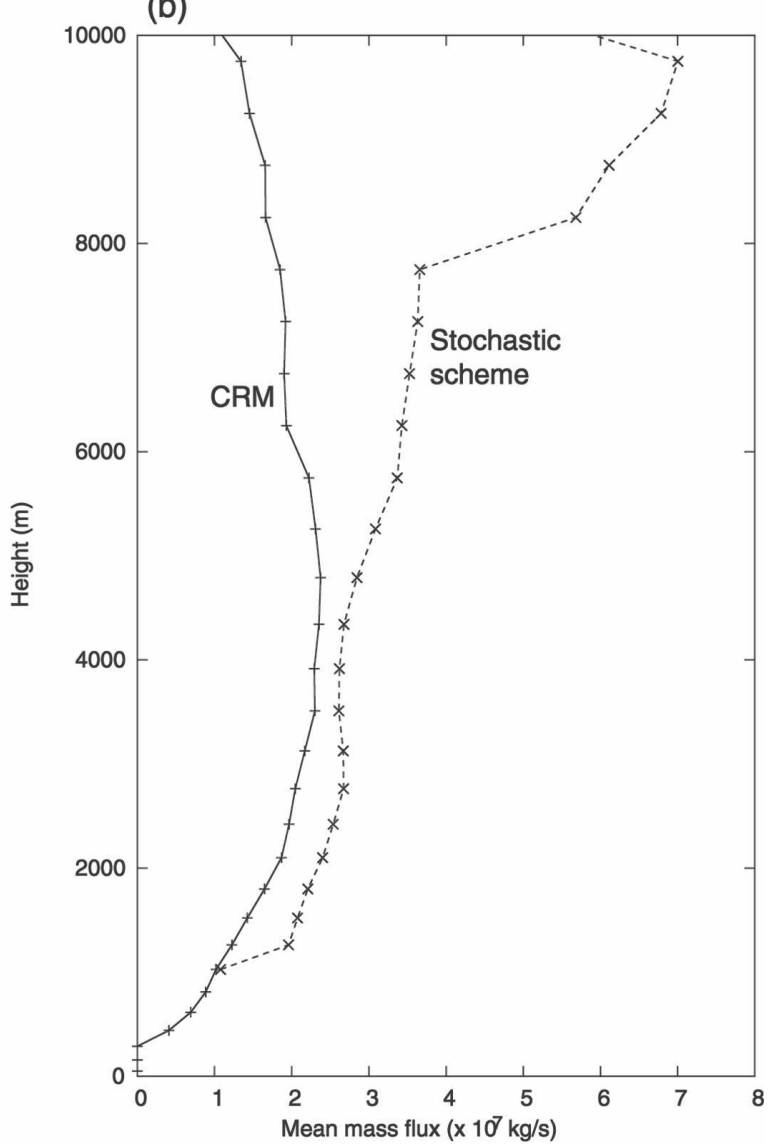

FIG. 8. (a) Histogram of individual plume mass fluxes at $5.75 \mathrm{~km}$ obtained at equilibrium in the SCM run with the stochastic parameterization over an area $(400 \mathrm{~km})^{2}$. Note the logarithmic scale. The mass flux is binned into intervals of $5 \times 10^{6} \mathrm{~kg} \mathrm{~s}{ }^{-1}$ and a line of best fit for an exponential distribution has been added. (b) The mean mass flux for each plume obtained from an exponential fit at each model level. Also shown is the mean mass flux of a convective updraft in the companion CRM simulation with a cooling rate of $8 \mathrm{~K} \mathrm{day}^{-1}$ (as in Fig. 3).

is therefore encouraging that an exponential distribution is, indeed, obtained at all heights, as shown for an example level in Fig. 8a.

The value of $\langle m\rangle$ obtained by fitting to the exponential distribution is shown as a function of height in Fig. $8 \mathrm{~b}$. As for the convective updrafts in the CRM, the ensemble-mean mass flux derived from the SCM varies little with height over much of the troposphere. Values in the lower-to-mid troposphere compare reasonably well to those in the CRM. Taken in conjunction with the mass-flux profiles of Fig. 6, this means that the SCM has fewer shallow clouds, but that there are a similar number of clouds at midlevels. This difference in the number of shallow clouds explains the difference in the total number of clouds seen in Table 2. The SCM also contains fewer clouds in the upper troposphere, but because the mass flux for each is larger (Fig. 8b) the total mass flux there agrees well with the CRM (Fig. 6). This upper-level behavior of $\langle m\rangle$ may reflect the ten- dency of plume models to overestimate mass fluxes at the level of zero buoyancy, as discussed by Kuang and Bretherton (2006). Moreover, the parameterization does not include downdrafts near cloud top, making it impossible to reproduce the overturning in convective anvils that occurs in the CRM.

\section{d. Tests of statistical fluctuations}

In this section, we consider fluctuations about the time-mean equilibrium SCM state, which arise from the quantization of convection into discrete plumes. A related issue is the steadiness of the forcing $\langle M\rangle$ provided by the CAPE closure since the closure is not a physical source of fluctuations. To investigate these issues, we compare the PDF of total mass flux from SCM experiments to the expected distribution, Eq. (2).

Some preliminary tests were conducted with an imposed closure: that is, with the ensemble-mean total mass flux $\langle M\rangle$ specified as a fixed value at a fixed ref- 
erence level. With a steady forcing imposed, the expected distribution should hold exactly and was, indeed, accurately reproduced (not shown).

We wish to check that any time step to time step variability in the closure calculated by the parameterization does not affect the convective statistics in an undesirable way. There are two aspects of the closure calculations to be considered: the closure time scale $T_{c}$ (section 4) and the profile averaging (step 1 of the algorithm in section $2 b$ ). Restrictions on the closure time scale arise independently of the averaging, limiting the choice of $k$ [Eq. (5)]. If $T_{c}$ is too short, then the parameterization will overstabilize the atmosphere. This will provoke the closure to reduce the forcing at future time steps and may result in undesirable on-off behavior. It is also important that $T_{c}$ not be too long, not least because the parameterization must be capable of responding to genuine, physical changes in the large-scale forcing. Our choice of $k$ necessarily represents a compromise and experimentation with the SCM indicates that $k=0.3 \mathrm{~s} \mathrm{~m}^{-1}$ (as in Table 1) provides a reasonable value. This corresponds to a closure time scale of a little over two hours in our SCM runs (Table 2), consistent with relaxation time scales used in other parameterizations (e.g., Betts and Miller 1986).

In an SCM, the only profile averaging available to provide a representation of the large-scale environment (section $2 \mathrm{~b}$ ) is time averaging. Thus, a complete definition of profile averaging for the stochastic parameterization must be deferred to future research and testing in a three-dimensional model. However, it is both possible and important to establish whether the sampling required to define a suitable large-scale environment ${ }^{7}$ is acceptable for practical use. The degree of sampling of individual profiles in order to compute a steady forcing must be compatible with the scales characterizing genuine variations in large-scale forcing.

Consider for example a typical global forecast model (or perhaps a high-resolution climate model) with a grid length of $64 \mathrm{~km}$. A practical number of profiles available for space-time averaging would be 150 , corresponding to an averaging area of side $\sim 320 \mathrm{~km}$ (the neighboring and next-to-neighboring grid boxes) and the six previous model time steps $(2 \mathrm{~h}$ with a 20 -min step). Running the SCM over an area of $(64 \mathrm{~km})^{2}$ and averaging the input profiles for the closure calculations over the previous 150 time steps is sufficient to produce the steady forcing required. Indeed, Fig. 9a indicates

\footnotetext{
${ }^{7}$ In SCM tests using the instantaneous profiles as input to the closure calculations, the computed $\langle M\rangle$ varied strongly between time steps and was the dominant source of convective fluctuations.
}

that a smaller sample size of 100 would also be acceptable.

Figure $9 \mathrm{~b}$ shows results for a larger grid length of 96 $\mathrm{km}$. Using the same space-time-averaging scales as above and assuming a longer model time step of $30 \mathrm{~min}$, the corresponding number of individual profiles is reduced to 44 (or to 30 if using 100 samples on the $64 \mathrm{~km}$ grid). This remains a sufficient number because of the smaller variability between profiles. We have also explicitly tested the profile averaging for smaller grid lengths more typical of a NWP model. The variability between individual profiles is then relatively large, but the increased number of profiles for the same spacetime averaging appears to provide more than adequate compensation.

\section{Conclusions}

There are strong theoretical and practical motivations for the use of stochastic convective parameterizations in both NWP and climate models, but work is only beginning to determine an appropriate representation of the variability. This paper has described a scheme in which the variability is designed to replicate the known fluctuations that occur about a state of radiativeconvective equilibrium above a uniform surface. The parameterization is based on an ensemble of entraining/detraining plumes with a conventional mass-flux closure. A Poisson distribution is expected for the occurrence of plumes with the plume mass fluxes (at any fixed level) being drawn from an exponential distribution (Craig and Cohen 2006). The variability arises in a natural way from the limited random sampling of the plume ensemble in each grid box.

The particular closure assumption and plume model used are adapted from the Kain-Fritsch (KF) parameterization. Note, however, that the various aspects of the stochastic parameterization have some independence so that one could derive new schemes of this general type (section $2 b$ ) by replacing, for example, the PDF or the plume model used.

Single-column tests have been carried out to establish that the parameterization is functioning as designed. In particular, the following expected behaviors are present:

1) In the limit of a large grid box, when the plume ensemble is well sampled, convective variability becomes small, and the parameterization approximates a deterministic scheme.

2) Mean profiles of temperature and humidity are comparable to those obtained in companion CRM simulations and to those in the SCM when a conventional parameterization $(\mathrm{KF})$ is used. 
(a)

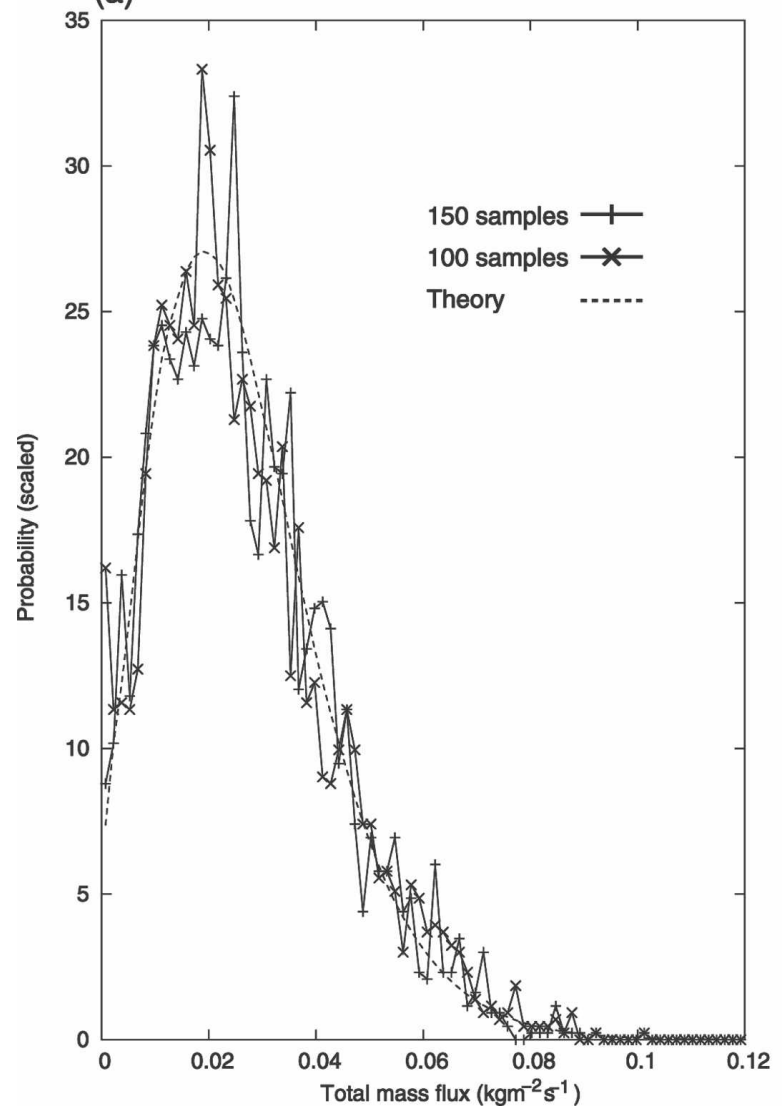

(b)

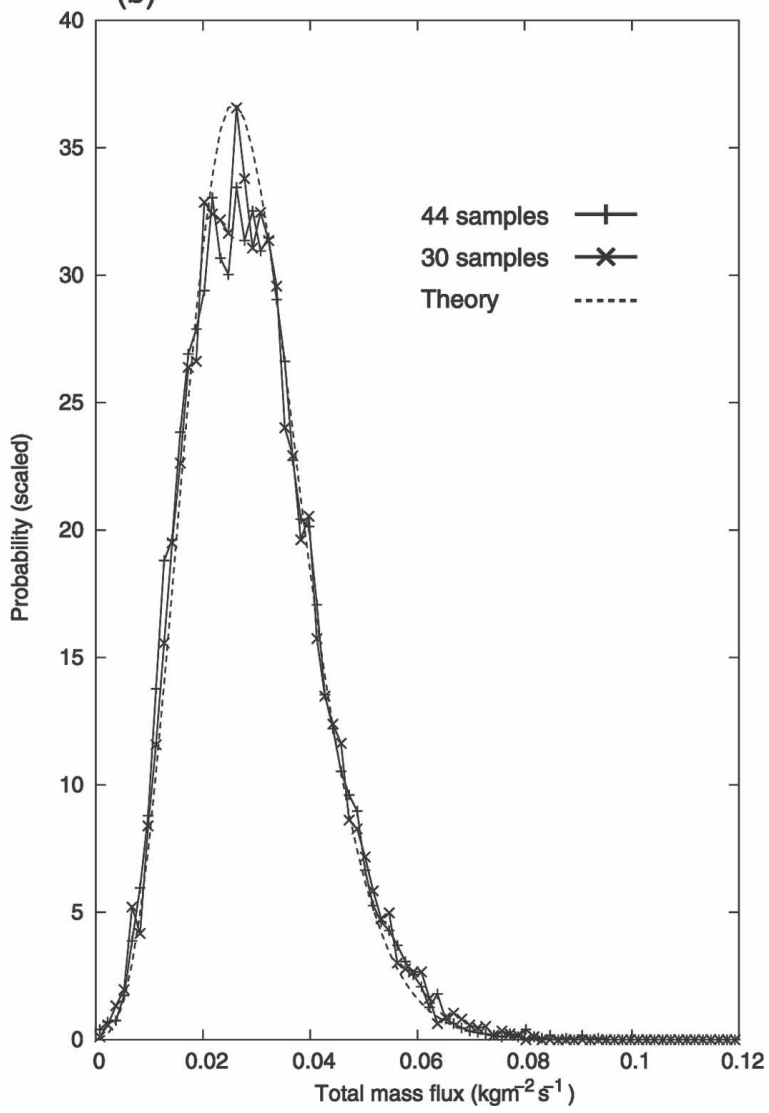

FIG. 9. Probability distribution functions at equilibrium for the total convective mass flux per unit area. Results from SCM runs are shown over (a) an area $(64 \mathrm{~km})^{2}$ and (b) an area $(96 \mathrm{~km})^{2}$. The profiles used for the closure calculations are determined from an average over previous time steps, the number of which is marked on the figure. Mass fluxes per unit area are recorded between the 40th and 80th days and binned into intervals of $0.0015 \mathrm{~kg} \mathrm{~m}^{-2} \mathrm{~s}^{-1}$. Also shown are the theoretical predictions from Eq. (2) for constant $\langle M\rangle$ (dotted line). The plots have been normalized such that the area under each PDF is unity.

3) Consistent with CRM simulations, the plume ensemble in the SCM exhibits an exponential distribution of mass fluxes above cloud base, with a mean mass flux that is approximately constant with height.

4) The prescribed distribution of total mass flux [Eq. (2)] is maintained in SCM experiments for columns of varying sizes.

5) An appropriate mean state for the closure calculation is produced by time averaging the atmospheric profile. The averaging interval used in this study was chosen conservatively in order to demonstrate the viability of the parameterization. A detailed examination of profile averaging strategies is not possible in the SCM context since the trade-off between temporal and spatial averaging cannot be explored.

Acknowledgments. R. Plant acknowledges funding from the UWERN programme, supported by NERC. We are grateful to $\mathrm{B}$. Cohen for providing us with data from her CRM simulations, and to J.-I. Yano and an anonymous reviewer for useful suggestions on the manuscript.

\section{APPENDIX}

\section{The Kain-Fritsch Parameterization}

The Kain-Fritsch convective parameterization has a long history, starting with the Fritsch and Chappell (1980) parameterization, significantly modified to produce the Kain and Fritsch $(1990,1993)$ parameterization and incrementally modified since. Kain (2004) recently discussed the version used in a semioperational configuration of the NCEP Eta Model. This version provided a starting point for the plume model and CAPE-closure code used in the stochastic parameterization. A brief description of it is presented here. It is suggested that the reader who requires a fuller descrip- 
tion consult section 2 of Kain et al. (2003) before studying some of the more technical material in the original papers.

The KF parameterization is a mass-flux scheme that may be divided into three parts. First, a decision is made as to whether convection will take place (the trigger). Second, the convection is described in terms of mass-flux profiles for a moist updraft, a moist downdraft, and compensating dry vertical motions. Third, the intensity of the convection must be determined (the closure).

The viability of convection is tested by constructing potential updraft source layers, each of which has thermodynamic properties computed as a mass-weighted average over several model levels. A parcel from the layer is assigned a temperature perturbation ${ }^{8} \delta T$ and its buoyancy is tested at the LCL. If buoyant, an initial vertical velocity is assigned. ${ }^{9}$ The second part of the scheme is then used to calculate the resulting updraft. The scheme searches for an updraft source layer that will engender deep convection. ${ }^{10}$ It considers first a layer based at the surface and, if necessary, the layer base is then incremented in steps of one model level up to $300 \mathrm{mb}$ above the surface. Should no suitable source layer be found for deep convection, then the source layer corresponding to the deepest cloud will, nonetheless, be used as a source for shallow convection. In the second and third parts of the parameterization, shallow convection differs in that (i) the detrainment profiles within the updraft are modified for consistency with LES results, (ii) it does not support an associated downdraft, and (iii) a different closure and lifetime is used. We retain the two types of convection in our stochastic parameterization but do not make the distinction (iii).

Updrafts are computed with an entraining/detraining plume model that describes two-way mass exchange between the updraft and its environment on each model level. Mixtures of updraft and environmental air are entrained or detrained according to whether they are positively or negatively buoyant, respectively. Such calculations require estimates of the maximum rate of entrainment ${ }^{11}$ and the distributions of environmental and updraft air in the mixtures (Kain and Fritsch 1990, their

\footnotetext{
${ }^{8}$ Related to the grid-scale vertical velocity at the LCL [Kain 2004, his Eq. (1)].

${ }^{9}$ Related to the temperature perturbation [Kain 2004, his Eq. (3)].

${ }^{10}$ That is, with a cloud depth exceeding a threshold value that is a function of the LCL temperature [Kain 2004, his Eq. (7)].

${ }^{11}$ The maximum entrainment rate is inversely proportional to the updraft radius [Kain and Fritsch 1990, their Eq. (1)], which is itself variable within the range 1-2 km [Kain 2004, his Eq. (6)] according to the grid-scale vertical velocity at the LCL.
}

section $2 \mathrm{~b}$ ). Conversion of condensate to precipitation within the updraft is determined by an empirical formulation [Kain and Fritsch 1990, their Eq. (9)]. The downdraft is initiated $150 \mathrm{mb}$ above the top of the updraft source layer. This is computed by assuming a fixed entrainment rate of environmental air above the updraft source layer and detrainment within and below. The downdraft is saturated above cloud base and dried by $20 \%$ relative humidity per kilometer below (Kain 2004, p. 178). Compensating vertical motions within the column are determined from mass continuity [Fritsch and Chappell 1980, their Eq. (10)].

A starting point for the above mass-flux calculations is the updraft mass flux at cloud base. This is guessed. Closure of the scheme consists of rescaling that guess (and all other mass fluxes appropriately) in an iterative manner until the desired intensity of convection is achieved. The intensity is defined by the requirement that the convection acts to remove at least $90 \%$ of the dilute CAPE within the closure time scale, $T_{c}$. Dilute CAPE is calculated for the entraining/detraining plume rather than for undilute parcel ascent. Note that, once convective activity is identified at a grid box, it persists for multiple time steps. Tendencies are applied to the grid box state over the time $T_{c}$ and the parameterization is not called again during this time; $T_{c}$ is set from an estimate of the transit time for a cloud to cross the grid box, subject to bounding values of 30 and $60 \mathrm{~min}$ (Fritsch and Chappell 1980, p. 1724). In our stochastic parameterization, the concept of a plume lifetime is retained (section 3a) but convective activity does not preclude the generation of other plumes during this time.

\section{REFERENCES}

Arakawa, A., 2004: The cumulus parameterization problem: Past, present, and future. J. Climate, 17, 2493-2525.

— ensemble with the large-scale environment, Part I. J. Atmos. Sci., 31, 674-701.

Betts, A. K., and M. J. Miller, 1986: A new convective adjustment scheme. Part II: Single-column tests using GATE wave, BOMEX, ATEX and arctic air-mass datasets. Quart. J. Roy. Meteor. Soc., 112, 693-709.

Bretherton, C. S., and P. K. Smolarkiewicz, 1989: Gravity waves, compensating subsidence and detrainment around cumulus clouds. J. Atmos. Sci., 46, 740-759.

Bright, D. R., and S. L. Mullen, 2002: Short-range ensemble forecasts of precipitation during the southwest monsoon. Wea. Forecasting, 17, 1080-1100.

Buizza, R., 1997: Potential forecast skill of ensemble prediction and spread and skill distributions of the ECMWF ensemble prediction system. Mon. Wea. Rev., 125, 99-119.

— 
of model uncertainties in the ECMWF ensemble prediction system. Quart. J. Roy. Meteor. Soc., 125, 2887-2908.

- P. L. Houtekamer, Z. Toth, G. Pellerin, M. Wei, and Y. Zhu, 2005: A comparison of the ECMWF, MSC, and NCEP global ensemble prediction systems. Mon. Wea. Rev., 133, 10761096.

Cohen, B. G., 2001: Fluctuations in an ensemble of cumulus clouds. Ph.D. thesis, University of Reading, 165 pp.

— , and G. C. Craig, 2004: The response time of a convective cloud ensemble to a change in forcing. Quart. J. Roy. Meteor. Soc., 130, 933-944.

- and - 2006: Fluctuations in an equilibrium convective ensemble. Part II: Numerical experiments. J. Atmos. Sci., 63, 2005-2015.

Craig, G. C., and B. G. Cohen, 2006: Fluctuations in an equilibrium convective ensemble. Part I: Theoretical formulation. $J$. Atmos. Sci., 63, 1996-2004.

Cullen, M. J. P., 1993: The unified forecast/climate model. Meteor. Mag., 122, 89-94.

Done, J. M., G. C. Craig, S. L. Gray, P. A. Clark, and M. E. B. Gray, 2006: Mesoscale simulations of organized convection: Importance of convective equilibrium. Quart. J. Roy. Meteor. Soc., 132, 737-756.

Donner, L. J., 1993: A cumulus parameterization including mass fluxes, vertical momentum dynamics, and mesoscale effects. J. Atmos. Sci., 50, 889-906.

Emanuel, K. A., 1994: Atmospheric Convection. Oxford University Press, $580 \mathrm{pp}$.

—_ and M. Bister, 1996: Moist convective velocity and buoyancy scales. J. Atmos. Sci., 53, 3276-3285.

Evans, R. E., M. S. J. Harrison, R. J. Graham, and K. R. Mylne, 2000: Joint medium-range ensembles from the Met Office and ECMWF systems. Mon. Wea. Rev., 128, 3104-3127.

Ewald, B., C. Penland, and R. Temam, 2004: Accurate integration of stochastic climate models with application to El Niño. Mon. Wea. Rev., 132, 154-164.

Frank, W. M., and C. Cohen, 1985: Properties of tropical cloud ensembles estimated using a cloud model and an observed updraft population. J. Atmos. Sci., 42, 1911-1928.

Fritsch, J. M., and C. F. Chappell, 1980: Numerical prediction of convectively driven mesoscale pressure systems. Part I: Convective parameterization. J. Atmos. Sci., 37, 1722-1733.

Grant, A. L. M., and A. R. Brown, 1999: A similarity hypothesis for shallow-cumulus transports. Quart. J. Roy. Meteor. Soc., 125, 1913-1936.

Gregory, D., and P. R. Rowntree, 1990: A mass flux convection scheme with representation of cloud ensemble characteristics and stability-dependent closure. Mon. Wea. Rev., 118, 14831506.

Guichard, F., and Coauthors, 2004: Modelling the diurnal cycle of deep precipitating convection over land with cloud-resolving models and single-column models. Quart. J. Roy. Meteor. Soc., 130, 3139-3172.

Hermanson, L., 2006: Stochastic physics: A comparative study of parameterized temperature tendencies in a global atmospheric model. Ph.D. thesis, University of Reading, 145 pp.

Horinouchi, T., and Coauthors, 2003: Tropical cumulus convection and upward-propagating waves in middle-atmospheric GCMs. J. Atmos. Sci., 60, 2765-2782.

Hou, D., E. Kalnay, and K. K. Droegemeier, 2001: Objective verification of the SAMEX '98 ensemble forecasts. Mon. Wea. Rev., 129, 73-91.
Houtekamer, P. L., L. Lefaivre, J. Derome, H. Ritchie, and H. L. Mitchell, 1996: A system simulation approach to ensemble prediction. Mon. Wea. Rev., 124, 1225-1242.

Kain, J. S., 2004: The Kain-Fritsch convective parameterization: An update. J. Appl. Meteor., 43, 170-181.

__, and J. M. Fritsch, 1990: A one-dimensional entraining/ detraining plume model and its application in convective parameterization. J. Atmos. Sci., 47, 2784-2802.

_, and 1 1993: Convective parameterization in mesoscale models: The Kain-Fritsch scheme. The Representation of $\mathrm{Cu}$ mulus Convection in Numerical Models, Meteor. Monogr., No. 46, Amer. Meteor. Soc., 165-170.

— - M. E. Baldwin, and S. J. Weiss, 2003: Parameterized updraft mass flux as a predictor of convective intensity. Wea. Forecasting, 18, 106-116.

Khouider, B., A. J. Majda, and A. Katsoulakis, 2003: Coarse grained stochastic models for tropical convection and climate. Proc. Natl. Acad. Sci. USA, 100, 11 941-11 946.

Kuang, Z., and C. S. Bretherton, 2006: A mass-flux scheme view of a high-resolution simulation of a transition from shallow to deep cumulus convection. J. Atmos. Sci., 63, 1895-1909.

LeMone, M. A., and E. J. Zipser, 1980: Cumulonimbus vertical velocity events in GATE. Part I: Diameter, intensity and mass flux. J. Atmos. Sci., 37, 2444-2457.

Lennard, R., 2004: The distribution of cumulus cloud sizes. M.S. thesis, Department of Meteorology, University of Reading, $67 \mathrm{pp}$.

Lin, J. W.-B., and J. D. Neelin, 2000: Influence of a stochastic moist convective parameterization on tropical climate variability. Geophys. Res. Lett., 27, 3691-3694.

— , and — 2002: Considerations for stochastic convective parameterization. J. Atmos. Sci., 59, 959-975.

$\longrightarrow$, and _ 2003: Toward stochastic deep convective parameterization in general circulation models. Geophys. Res. Lett., 30, 1162, doi:10.1029/2002GL016203.

Majda, A. J., and B. Khouider, 2002: Stochastic and mesoscopic models for tropical convection. Proc. Natl. Acad. Sci. USA, 99, 1123-1128.

Mapes, B. E., 1997: Equilibrium vs. activation control of largescale variations of tropical deep convection. The Physics and Parameterization of Moist Atmospheric Convection, R. K. Smith, Ed., Kluwer Academic, 321-358.

Mylne, K. R., R. E. Evans, and R. T. Clark, 2002: Multi-model multi-analysis ensembles in quasi-operational medium-range forecasting. Quart. J. Roy. Meteor. Soc., 128, 361-384.

Nober, F. J., 2003: Development of a convective cloud field model based upon principles of self-organization. Ph.D. thesis, University of Hamburg, 109 pp.

Ooyama, K., 1971: A theory on parameterization of cumulus convection. J. Meteor. Soc. Japan, 49, 744-756.

Palmer, T. N., 2001: A nonlinear dynamical perspective on model error: A proposal for non-local stochastic-dynamic parametrization in weather and climate prediction models. Quart. J. Roy. Meteor. Soc., 127, 279-304.

Penland, C., 2003: A stochastic approach to nonlinear dynamics: A review. Bull. Amer. Meteor. Soc., 84, ES43-ES52.

Peters, O., and J. D. Neelin, 2006: Critical phenomena in atmospheric precipitation. Nature Phys., 2, 393-396.

Press, W. H., S. A. Teukolsky, W. T. Vetterling, and B. P. Flannery, 1992: Numerical Recipes in Fortran 77: The Art of Sci- 
entific Computing. 2nd ed. Cambridge University Press, 934 pp.

Ricciardulli, L., and R. R. Garcia, 2000: The excitation of equatorial waves by deep convection in the NCAR Community Climate Model (CCM3). J. Atmos. Sci., 57, 3461-3487.

Robe, F. R., and K. A. Emanuel, 1996: Moist convective scaling: Some inferences from three-dimensional cloud ensemble simulations. J. Atmos. Sci., 53, 3266-3275.

Shutts, G. J., and M. E. B. Gray, 1999: Numerical simulations of convective equilibrium under prescribed forcing. Quart. J. Roy. Meteor. Soc., 125, 2767-2787.

Smith, R. N. B., 1990: A scheme for predicting layer clouds and their water content in a general circulation model. Quart. J. Roy. Meteor. Soc., 116, 435-460.

— cesses. Met Office Tech. Rep., Unified Model Documentation Paper 24, model version 4.5, 118 pp.

— D. Gregory, J. F. B. Mitchell, A. C. Bushell, and D. R. Wilson, 1998: Large-scale precipitation. Met Office Tech. Rep., Unified Model Documentation Paper 26, model version 4.5, $38 \mathrm{pp}$.

Stensrud, D. J., J.-W. Bao, and T. T. Warner, 2000: Using initial condition and model physics perturbations in short-range ensemble simulations of mesoscale convective systems. Mon. Wea. Rev., 128, 2077-2107.

Teixeira, J., and C. A. Reynolds, 2008: Stochastic nature of physical parameterizations in ensemble prediction: A stochastic convection approach. Mon. Wea. Rev., in press.
Wilks, D. S., 2005: Effects of stochastic parameterizations in the Lorenz '96 system. Quart. J. Roy. Meteor. Soc., 131, 389-407.

Williams, P. D., 2006: Modelling climate change: The role of unresolved processes. Philos. Trans. Roy. Soc. London, 363, 2931-2946.

Wilson, D. R., and S. P. Ballard, 1999: A microphysically based precipitation scheme for the UK meteorological office unified model. Quart. J. Roy. Meteor. Soc., 125, 1607-1636.

Xu, K.-M., A. Arakawa, and S. K. Krueger, 1992: The macroscopic behavior of cumulus ensembles simulated by a cumulus ensemble model. J. Atmos. Sci., 49, 2402-2420.

Yang, Z. W., and R. W. Arritt, 2002: Tests of a perturbed physics ensemble approach for regional climate modeling. J. Climate, 15, 2881-2896.

Yano, J.-I., K. Fraedrich, and R. Blender, 2001: Tropical convective variability as $1 / f$ noise. J. Climate, 14, 3608-3616.

_, R. Blender, C. Zhang, and K. Fraedrich, 2004a: 1/f noise and pulse-like events in the tropical atmospheric surface variabilities. Quart. J. Roy. Meteor. Soc., 130, 1697-1721.

_ , F. Guichard, J.-P. Lafore, J.-L. Redelsperger, and P. Bechtold, 2004b: Estimations of mass fluxes for cumulus parameterizations from high-resolution spatial data. J. Atmos. Sci., 61, 829-842.

Zhang, F., C. Snyder, and R. Rotunno, 2003: Effects of moist convection on mesoscale predictability. J. Atmos. Sci., 60, $1173-1185$. 